\title{
Images Re-vues
}

Histoire, anthropologie et théorie de l'art

19 | 2021

Images scientifiques / images artistiques:

croisements méthodologiques

\section{Raisonner sur des images. Restaurer la Sainte-Anne.}

Reasoning on Pictures, Restoring the Saint Anne

Pierre Leveau

\section{CpenEdition}

\section{Journals}

Édition électronique

URL : https://journals.openedition.org/imagesrevues/9684

DOI : 10.4000/imagesrevues.9684

ISSN : 1778-3801

Éditeur :

Centre d'Histoire et Théorie des Arts, Groupe d'Anthropologie Historique de l'Occident Médiéval, Laboratoire d'Anthropologie Sociale, UMR 8210 Anthropologie et Histoire des Mondes Antiques

\section{Référence électronique}

Pierre Leveau, «Raisonner sur des images. Restaurer la Sainte-Anne. », Images Re-vues [En ligne], 19 | 2021, mis en ligne le 30 juin 2021, consulté le 28 février 2022. URL : http://journals.openedition.org/ imagesrevues/9684; DOI : https://doi.org/10.4000/imagesrevues.9684

Ce document a été généré automatiquement le 28 février 2022.

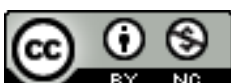

Images Re-vues est mise à disposition selon les termes de la Licence Creative Commons Attribution Pas d'Utilisation Commerciale 4.0 International. 
Raisonner sur des images. Restaurer la Sainte-Anne.

Reasoning on Pictures, Restoring the Saint Anne

Pierre Leveau

Introduction 
1 La restauration des images artistiques fait aujourd'hui appel à l'imagerie scientifique. Ces deux régimes iconographiques sont étroitement liés dans le champ de la conservationrestauration du patrimoine. Les œuvres sont souvent examinées sous différentes lumières ou analysées par de multiples techniques dans le cadre du diagnostic qui précède l'intervention. Ce processus, qui va du constat d'état aux préconisations après restauration, a été modélisé en 2013 par les professionnels du domaine ${ }^{1}$. Comment étudier les relations sémiotiques que nouent ces deux régimes iconographiques, scientifique et

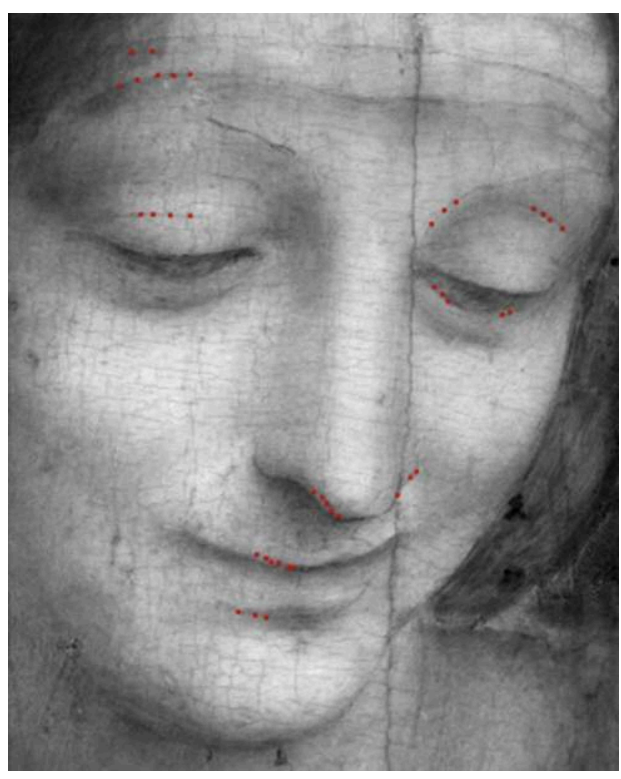
artistique? La restauration du dernier tableau de Léonard de Vinci, «La Vierge à l'Enfant avec Sainte Anne », est assez instructive à cet égard. L'opération commencée en 2008 dura quatre ans et s'acheva par une exposition $^{2}$. On produisit un documentaire pour l'occasion ${ }^{3}$ et une dizaine de cartons d'archives comprenant plus de 500 images de l'œuvre, accompagnées de graphiques et de tableaux. Cette documentation est la propriété du Département des peintures du musée du Louvre ${ }^{4}$ et du Centre de recherche et de restauration des musées de France qui l'assista ${ }^{5}$. Chaque acteur a archivé selon des méthodes différentes, la partie qu'il produisit, institutionnalisant ainsi le clivage entre images scientifiques et artistiques. Le Département des peintures conserve la documentation artistique à la Porte aux Lions et le Centre de recherche archive la sienne sur deux sites : le pavillon de Flore à Paris pour la partie scientifique et les petites écuries du Roi à Versailles pour la restauration $^{6}$. Comment ces différents types de clichés furent-ils utilisés et interprétés? Quels concepts mobiliser pour étudier leurs relations et leurs rapports à l'œuvre ? Comment suivre le fonctionnement sémiotique de la raison dans ce champ? On répond dans cette étude de cas en identifiant les raisonnements que les experts firent sur l'œuvre et ses images, puis en définissant les points de bascule d'un régime iconographique à l'autre: de l'artistique vers le scientifique et inversement. On les articule enfin en formulant une hypothèse sur cette bascule de point de vue, inspirée du cinéma.

\section{Images artistiques et scientifiques}


Figure 1

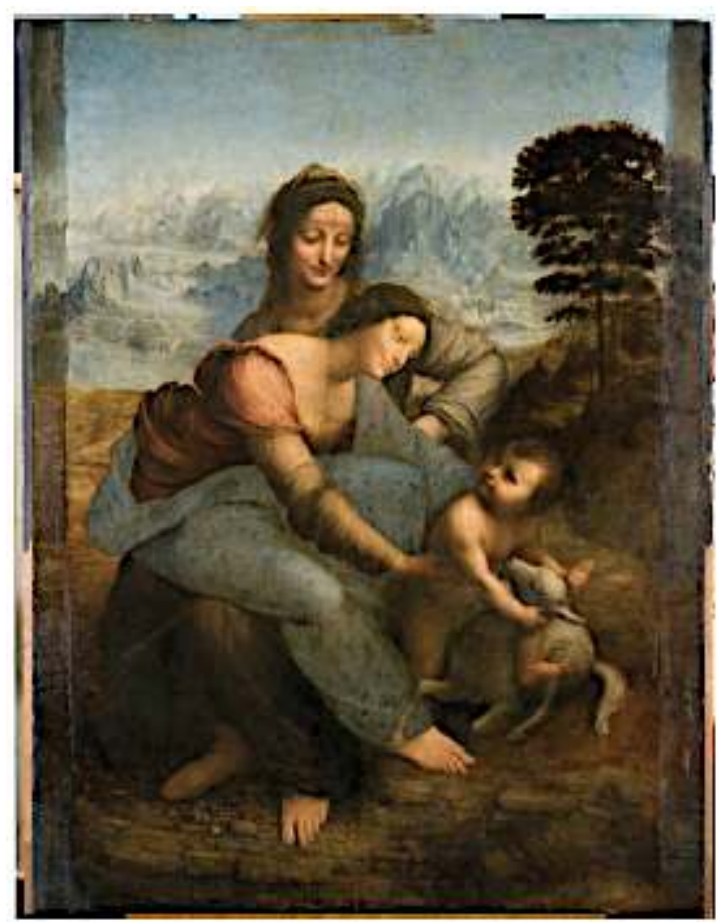

Tableau avant restauration (27380)

(C) $2 R M F$

2 Il apparut en 2008 que la Sainte-Anne devait être restaurée. Son vernis avait jauni et les repeints pris dans la couche protectrice tachaient l'image, dont on n'apercevait plus ni les reliefs ni le modelé des couleurs. L'état de conservation de l'œuvre était en revanche satisfaisant : hormis les fentes du support ${ }^{7}$, la couche picturale et la préparation avaient bien résisté au temps ${ }^{8}$. Seule l'image posait problème, parce qu'elle n'était plus compréhensible et indigne du génie de Léonard. Le jaunissement du vernis, ses chancis et les repeints occultaient l'œuvre et faussaient son interprétation. Les étudiants croyaient que le manteau de la Vierge était brodé ; le public averti ne parvenait plus à différencier les repeints et les repentirs de l'artiste; le tableau autorisait toutes les interprétations, que son incompréhensibilité rendait curieusement acceptables. Il fallait se rendre à l'évidence : l'œuvre était là, mais pas l'image artistique, qu'il fallait donc restaurer (Fig. 1).

L'opération se présentait dans ce contexte comme une enquête, une recherche où l'imagerie scientifique fut immédiatement convoquée. Il s'agissait de trouver les causes de la disparition de l'image, apparemment due au jaunissement du vernis et à ses chancis, tout en examinant l'état de la couche picturale et en ôtant les repeints. Le tableau avait déjà été restauré par le passé. Les dernières interventions en 1947, en 1953 puis en 1972, avaient été précédées d'un examen scientifique ${ }^{9}$. Elle fut à nouveau photographiée en lumière directe et rasante en 2008, sous binoculaire, ultraviolet et réflectographie infrarouge, dans son ensemble et en détail. Le laboratoire ajouta ainsi une centaine de clichés au dossier scientifique, déjà épais, de la Sainte-Anne ${ }^{10}$. Qu'apprirent-ils aux maîtres d'œuvre de l'opération? Comment les historiens d'art, les scientifiques et les conservateurs-restaurateurs interprétèrent-ils ces clichés? Quel rôle l'imagerie «scientifique » joua-t-elle dans la restauration? 


\section{Analogie}

Figure 2
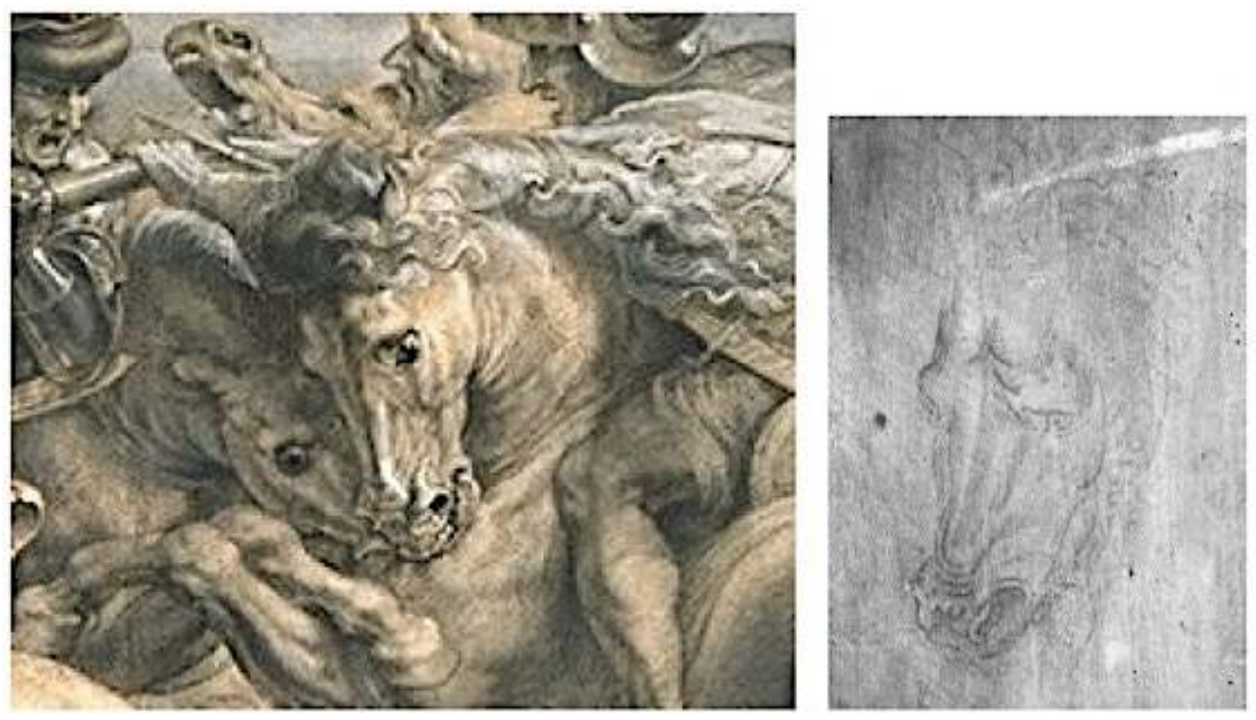

Détail de la tête de cheval esquissée au revers de la Sainte-Anne (16289) et Rubens, d'après Léonard, détail de la Bataille d'Anghiari (Paris, Louvre, INV 20271)

(c) $2 R M F$

le lancement de l'opération, lors du décadrage de l'œuvre au musée, une découverte inattendue rappela à tous les acteurs la place que les hommes et les machines occupaient dans le système socio-technique d'interprétation des images. Une légère modification de la texture des parties supérieures des planches du panneau retint l'attention d'un conservateur, Sylvain Laveissière ${ }^{11}$. Entre les halos d'anciennes taches d'humidité et les stries des outils de taille, l'expert discerna deux zones de traits dont l'orientation accrocha son regard. Leur déclinaison laissait supposer une intervention humaine, non naturelle et artistique plutôt qu'artisanale. Sur la deuxième planche du panneau, sous la traverse qui consolidait le support, le conservateur reconnut une tête de cheval, puis sur la troisième un demi-crâne humain (Fig. 2).

Fantasme ou réalité? Léonard s'intéressa comme on le sait aux phénomènes projectifs ${ }^{12}$. Pour interpréter la découverte et neutraliser les biais de la subjectivité, les experts demandèrent au laboratoire d'objectiver le phénomène par une série de clichés en réflectographie infrarouge. L'imagerie scientifique confirma l'existence des dessins et en révéla même un troisième. Sur la quatrième planche du panneau, la réflectographie infrarouge fit apparaittre quelques lignes esquissant la silhouette d'un enfant accroupi derrière un agneau couché. Ces trois dessins étaient-ils de la main du maître ? Toute une série d'analogies pouvait le faire croire. Chaque dessin ressemblait en effet à un autre déjà attribué à Léonard : la tête de cheval à une étude préparatoire de 1504 pour la Bataille d'Anghiari; le demi-crâne à une figure daté de 1489 ; l'esquisse de l'enfant à celle du Jésus peint de la Sainte-Anne ${ }^{13}$. Mais comparaison n'est pas raison et aucun historien d'art ne pouvait tenir ces analogies pour des preuves. On sait que Léonard demandait à ses assistants de copier ses travaux pour en suivre la composition. Ses élèves l'imitaient pour apprendre de lui les secrets de l'art et d'autres artistes reproduisirent plus tard ses œuvres pour s'en inspirer. Le Centre de documentation du 
département des peintures du musée du Louvre mettait régulièrement à jour le sousdossier des "analogies » de l'œuvre, qui répertoriait toutes les images similaires ou iconiquement liées à la Sainte-Anne ${ }^{14}$. Le fait que la tête du cheval de la Bataille d'Anghiari commença à être copiée dès 1526 bloqua dans un premier temps le processus d'authentification. Rien n'autorisait en effet à attribuer au maître le dessin découvert au revers, qui pouvait être l'œuvre d'un disciple. Les similitudes ouvraient le champ des possibles, sans livrer de conclusion nécessaire. En multipliant les interprétations, elles donnaient à penser, mais ne prouvaient rien, sinon la nécessité de douter. Le réseau des analogies de la Bataille d'Anghiari bloquaient l'abduction sensée remonter de l'œuvre à l'auteur et de l'effet à la cause.

\section{Abduction}

6 Aussi les conservateurs du département des peintures commandèrent-ils au Centre de recherche une nouvelle expertise pour trancher le débat. La réflectographie infrarouge avait déjà permis d'identifier des matériaux, mais pas de remonter de l'œuvre à l'auteur. Le fait que l'image apparaisse clairement sous infrarouge s'expliquait dès que l'on supposait que les figures aient été tracées au carbone, avec un fusain par exemple. L'abduction routinière pour les experts allait de l'image scientifique, considérée comme un effet, aux matériaux carbonés tenus pour sa cause. Un nouvel examen à la loupe binoculaire le confirma et permit d'exclure l'encre ou de la pierre noire, en raison de l'aspect sec et pulvérulent du trait évident sur les macrophotographies ${ }^{15}$. Les propriétés physiques des matériaux réduisaient les possibilités, par exclusions successives, mais ne permettaient pas d'authentifier les dessins, faute de remonter de l'outil à la main. Une étude stylistique aurait pu identifier leur auteur en comparant ces tracés à d'autres ; mais la rugosité du support, qui durcissait le trait, la rendait impraticable. Les experts se firent graphologues pour formuler une ultime conjecture. Les hachures du modelé de la tête de cheval et du demi-crâne n'inclinaient pas dans le même sens. Celles du cheval allaient de gauche à droite, mais celles du crâne en sens contraire. Si les premières pouvaient avoir été faites par un gaucher, les secondes auraient pu l'être par un droitier, rien n'interdisant de penser que l'inverse fut vrai, les lignes ayant pu être tirées de bas en haut et non de haut en bas. Parce-que Léonard était gaucher, sinon ambidextre, la non-congruence des tracés bloquait l'attribution des figures: elles n'étaient pas forcément de la même main et auraient pu être tracées par un droitier ou un gaucher, donc par Léonard lui-même ou un disciple imitant scrupuleusement le maitre. En quelque sens qu'on les prenne, les macrophotographies du nouvel examen ne permettaient donc pas d'authentifier les dessins du revers. Le passage de l'analogie à l'abduction augmentait comme précédemment le nombre d'interprétations au lieu de le réduire. Les conservateurs du Louvre et les scientifiques du Centre n'ayant pas d'autre indice à exploiter, la découverte « sérendépitique » fut jugée apocryphe, par défaut.

\section{Indices}

7 Que conclure de ce premier épisode sur le rapport des images scientifiques et artistiques dans le champ de la conservation-restauration? On pourrait penser que les critiques d'art raisonnent plutôt par analogie, tandis que les scientifiques procèdent par abduction, sans caractériser pour autant ces professions par ces modes d'inférence ${ }^{16}$. Mais le cas de la Sainte-Anne montre a contrario que les historiens d'art ne 
font pas vraiment d'analogies : ils les défont plutôt. Les contrefaçons sont si fréquentes dans le champ des arts que ce type de raisonnement ne peut qu'y susciter la méfiance. Les analogies entre peinture et dessin sont inévitables dans l'œuvre de Léonard, puisqu'il dessinait toutes ses compositions avant de peindre. Si un seul des trois cartons qu'il conçut pour la Sainte-Anne entre 1501 et 1508 nous est parvenu, les nombreuses études qu'il réalisa pour les composer ont été conservées. Ses élèves ont transmis des copies de l'œuvre en cours d'exécution et ses suiveurs ont reproduit les deux cartons aujourd'hui perdus. La centaine d'images que contient le sous-dossier des analogies de la Sainte-Anne autorise dans ces conditions un grand nombre de rapprochements, que l'on peut sommairement diviser en deux catégories. La première relie différents travaux d'un même genre - peintures ou dessins - tandis que la seconde regroupe ceux de genres différents. On peut marquer cette différence en parlant de similitude dans le premier cas et d'analogie au dans le second. On identifie dans les deux cas un point commun ; mais c'est une qualité dans le premier et une relation dans le second. Vincent Delieuvin a présenté, dans le catalogue de l'exposition, ce prodigieux réseau de ressemblances, en rappelant que le travail des historiens d'art ne consiste pas à multiplier les interprétations, mais à établir des faits pour comprendre la genèse d'une œuvre ${ }^{17}$.

Figure 3

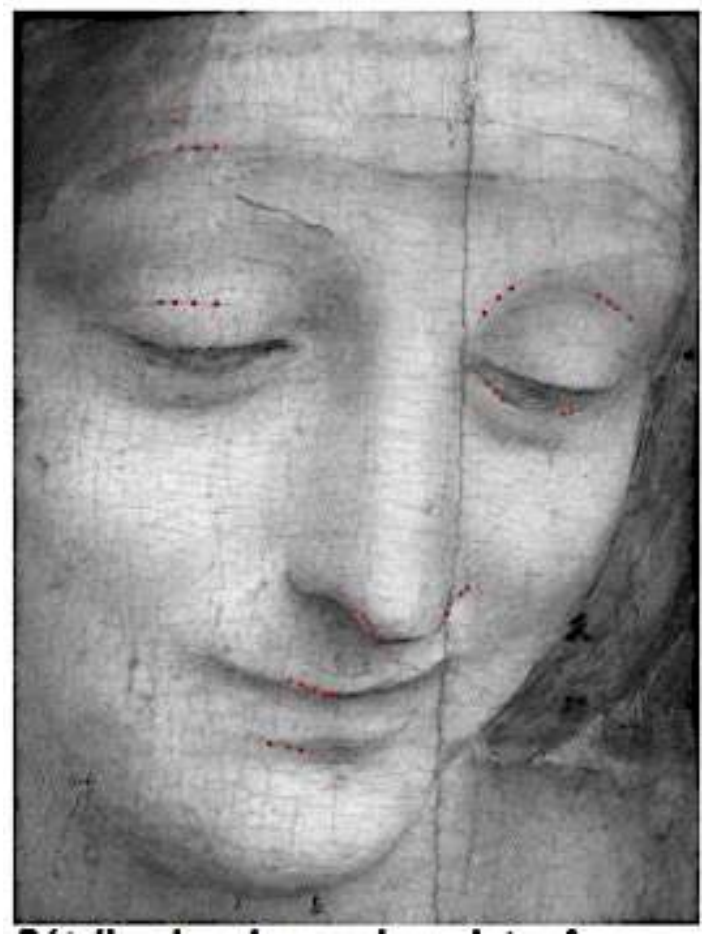

Détail du visage de sainte Anne en réflectographie IR situant les points de spolvero (16289) (c) $2 R M F$

8 Dans le cas de la Sainte-Anne, le point le plus important consistait à expliquer le passage du dessin à la peinture justifiant les analogies. Or ce type de point existe bien dans les œuvres de la Renaissance, où les peintres perçaient leur carton avant de l'appliquer sur le support à peindre et de déposer dans les trous une poudre de charbon de bois ou à les repasser à la pierre noire ou au fusain. Cette technique de transfert, appelée spolvero, 
permettait de reporter la composition du poncif sur le support à peindre en reliant par une ligne les points qui apparaissaient sur la préparation. Tous les historiens d'art supposaient que Léonard avait utilisé un poncif pour peindre la Sainte-Anne, sans pouvoir le prouver, puisque le carton correspondant avait disparu et que la couche picturale masquait les traces du spolvero. Les conservateurs du Louvre confirmèrent cette hypothèse en discernant, sur les réflectogrammes fournis par le Centre de recherche et de restauration, une série de points isolés mais reliés par des lignes, qui s'expliquaient si l'on supposait que Léonard avait eu utilisé un poncif (Fig. 3) ${ }^{18}$.

En raisonnant comme précédemment par abduction, sur la base d'indices matériels et non de relations iconiques, ils purent ainsi conjecturer le fait historique qui justifiait l'analogie. Les points du spolvero étaient la clef qui permettait de passer du dessin à la peinture et de reconstruire le processus créatif en s'en tenant aux faits. Véritables punctum du studium, ces points sont les indices matériels d'un changement de régime iconographique qui signalent le passage d'un type d'image à l'autre.

\section{Points de bascule}

10 Notre hypothèse sur le fonctionnement sémiotique de la raison dans le champ de la conservation-restauration sera donc la suivante: si les analogies des historiens d'art sont les indices qui leur permettent de raisonner par abduction, comme le font couramment les scientifiques, les activités de ces spécialistes diffèrent moins par leurs modes d'inférences que par leurs objets: des matériaux dans un cas, des œuvres dans l'autre. Cette hypothèse pourrait se formuler ainsi : le rapport des « historiens d'art » aux images artistiques est le même que celui des « scientifiques du patrimoine » à leurs propres images; tous raisonnent par abduction pour remonter d'un effet à une cause, de part et d'autre de leur frontière disciplinaire, jusqu'à un auteur dans un cas ou à ses matériaux dans l'autre. Mais on jugerait mal la situation si l'on s'en tenait là. Le propre de la conservation-restauration du patrimoine, considérée comme un domaine d'activité interprofessionnel normalisée ${ }^{19}$, est en effet d'amener ces experts à croiser leurs interprétations. L'historien d'art interprète l'imagerie scientifique qui ne relève pas de sa discipline, comme le scientifique examine, avec ses propres outils, les images artistiques étrangères à la sienne. L'objet à conserver et à restaurer se constitue dans le croisement de leurs échanges interprofessionnels. Compte-tenu de cette spécificité, notre hypothèse de travail sera donc plutôt la suivante : le rapport des historiens d'art aux images scientifiques est finalement le même que celui des scientifiques du patrimoine aux images artistiques. Tous y recherchent des indices autorisant des conjectures par abduction ou des preuves réduisant leur nombre par réfutation. Notre enquête portera dans ces conditions sur la communication entre ces professions et sur l'articulation de leurs domaines d'investigations respectifs et de leurs régimes iconographiques. Comment chacun accède-t-il à l'univers de l'autre depuis le sien? Quel point les $y$ fait basculer et comment ce basculement s'opère-t-il ?

\section{Points de Lyncée}




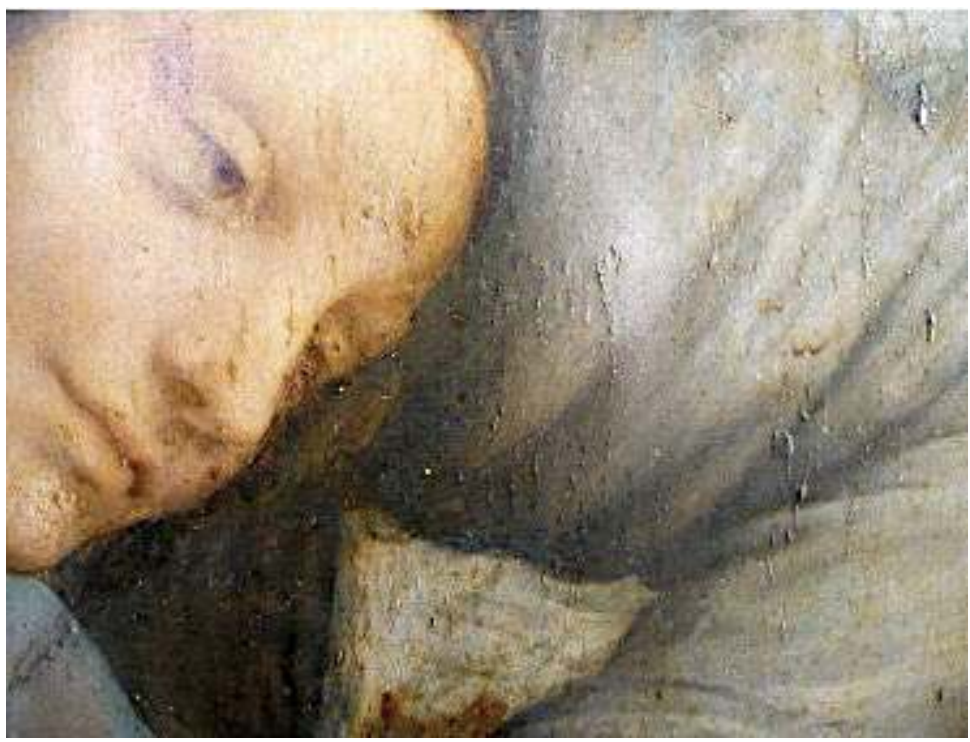

Détail des soulèvements le long des fibres verticales du bois (27379)

CC2RMF

11 L'étude dendrochronologique du panneau et des traverses, commandée par les conservateurs du musée au Centre de recherche permet de préciser ce point ${ }^{20}$. On y voit se succéder les raisonnements précédemment évoqués, hormis celui par analogie auquel les experts ont rarement recours parce qu'il ne prouve rien. C'est justement ce qui le rend intéressant. La question posée par les conservateurs portait classiquement sur l'analyse et la datation du support. Son vieillissement et les contraintes exercées par les traverses au revers posaient en effet problème. Quels effets avaient-elles sur la couche picturale et donc sur l'image? Comment neutraliser les plus indésirables ? La conservatrice-restauratrice qui dressa le constat d'état de l'œuvre, Nathalie Le Dantec, avait déjà répondu en 2006 à ces questions et formulé une série de préconisations que l'étude dendrochronologique pouvait étayer ${ }^{21}$. Son œil exercé avait repéré deux types d'altérations de la couche picturale, qu'elle attribua à des causes distinctes en raison de leur emplacement et de leur aspect. Les unes, en forme de cloques, s'alignaient verticalement sur la partie bombée de la troisième planche, tandis que les autres, en saillie, apparaissaient sur l'ensemble du tableau (Fig. 4).

Considérant que l'apparition de craquelures en tuile et en écaille pouvait respectivement s'expliquer par la rétractation du bois et par un défaut de séchage du liant, la conservatrice-restauratrice attribua les premières aux mouvements du support et les secondes à d'anciennes retouches. La perception de ces altérations déclencha comme précédemment une abduction remontant de l'effet à la cause qui changea le régime iconographique de l'œuvre. La focalisation sur le punctum, c'est-à-dire sur l'indice matériel, au détriment du sujet, i.e. du studium, donna à l'image un statut scientifique et non plus artistique. Le tableau n'est plus à cet instant le support d'un jugement de goût, mais de connaissance. De la vue d'ensemble au détail, le changement de focale induit par la perception de l'indice fit surgir l'histoire matérielle de l'œuvre, en deçà de celle qu'elle narrait ${ }^{22}$. L'experte conjectura à l'œil nu et par abduction les causes profondes des altérations perceptibles en surface. On pourrait appeler « point de Lyncée » ce punctum, où l'on bascule de l'image artistique à l'image scientifique ou du 
jugement esthétique au jugement de connaissance et de l'œuvre à l'indice. Lyncée personnifiait l'acuité visuelle dans la mythologie grecque et fut la vigie de l'Argos ${ }^{23}$; comme l'œil du héros voyait l'ennemi caché sous terre ou derrière les brumes, celui de l'experte perçut les causes de l'altération de l'image sous la couche picturale.

Figure 5

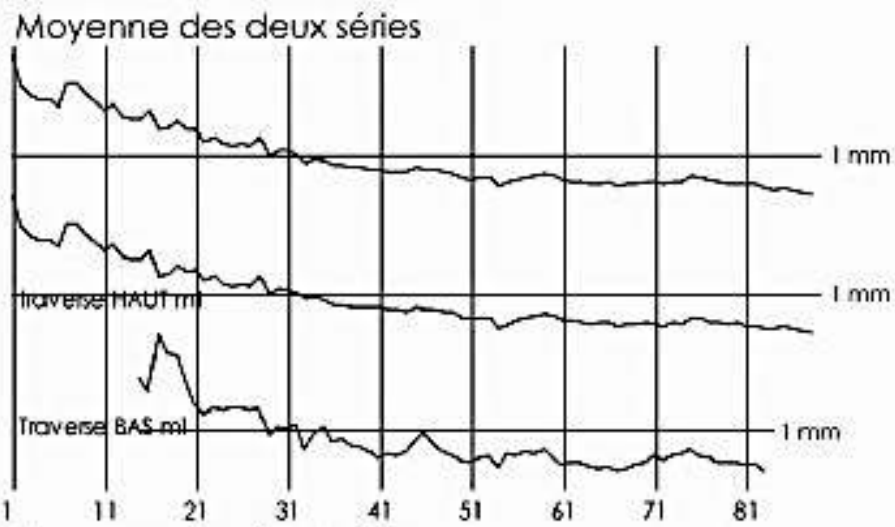

Moyenne des deux séries

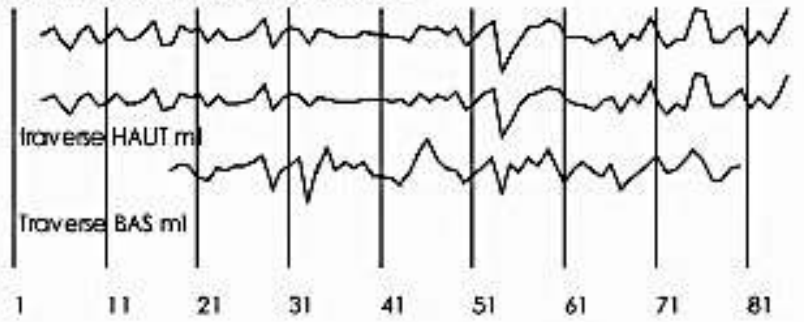

Synchronisation des séries dendrochronologiques des traverses (16287) (c) $2 R M F$

L'examen dendrochronologique commandé en 2008 par les conservateurs du Louvre, confirma-t-il la vue de la conservatrice-restauratrice? L'étude ne permit pas à l'analyste, Catherine Lavier, de dater les traverses et les planches du support. Identifier dendrochronologiquement un bois consiste à comparer son profil de croissance aux modèles de la même essence connus dans une région, à une époque et dans un climat donné, puis à évaluer son degré de ressemblance et à en tirer des conclusions sur sa provenance et son histoire. L'identification s'apparente, dans ces conditions, à un raisonnement par prototype basé sur des corrélations statistiques. Dans le cas de la Sainte-Anne, le montage qui occultait le revers interdisait l'examen d'une partie des traverses et la présence d'un nœud sur celle du bas diminuait la quantité d'information exploitable. La comparaison des profils obtenus permit cependant à l'analyste d'établir que les traverses provenaient d'arbres différents, mais contemporains (Fig. 5). 
Figure 6

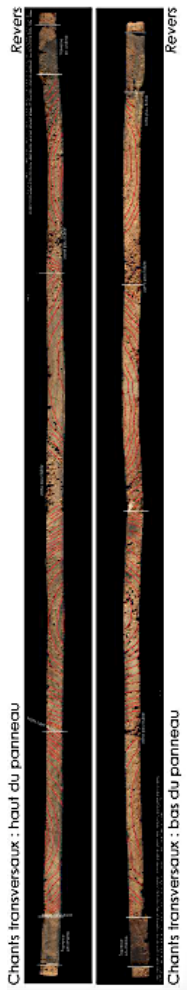

Restitution photographique des chants transversaux du panneau (16287)

(C) $2 R M F$

14 Leurs profils de croissance ne se correspondaient pas, mais certains points de leurs courbes attestaient leur synchronisation. Les tests de corrélation avec des séries chronologiques tirées de l'archéologie du bâti, des matériaux de fouilles et de la lutherie, s'avérèrent peu probants: les années qui ressortirent, 1505 et 1838, correspondaient aux hypothèses à départager, si bien que l'examen dendrochronologique ne permit pas de trancher le débat sur l'authenticité des traverses. A contrario, la restitution photographique des chants transversaux des planches $\mathrm{du}$ panneau et leur étude dendrométrique justifia l'abduction de la conservatrice-restauratrice (Fig. 6).

Figure 7
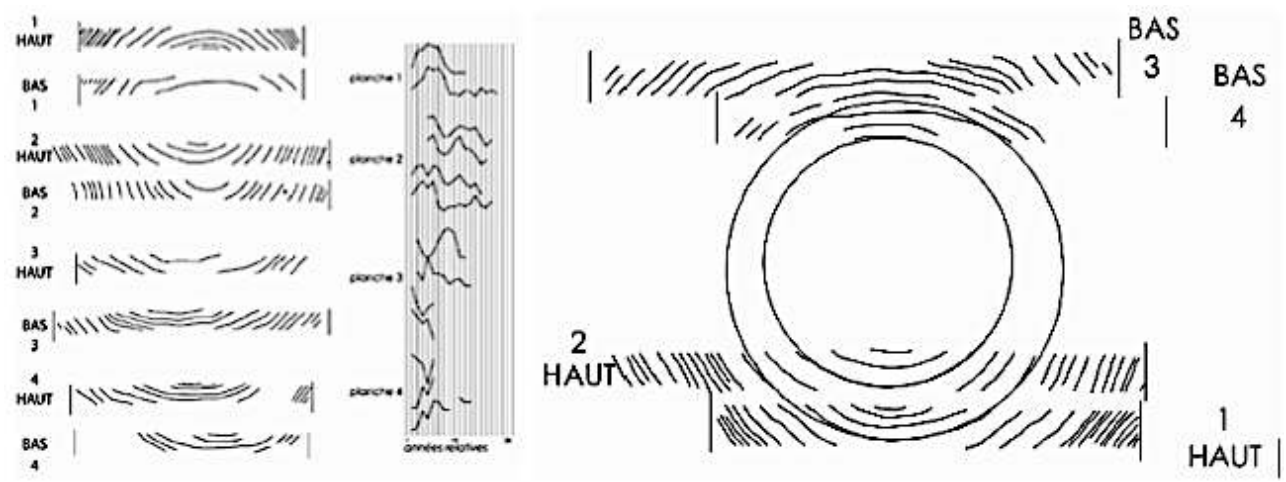

Largeur des cernes et reconstitution de la grume (16287)

(C) $2 R M R$ 
L'angle des cernes de croissance par rapport à la coupe indiquait en effet un débit sur dosse, tangentielle à la grume. Les planches avaient été sciées parallèlement à l'axe du tronc, par traits successifs. La largeur comparable des cernes et la synchronisation des séries laissaient supposer que les quatre planches provenaient, si non de la même grume, du moins du même arbre. Les règles du débit sur dosse étant connues ${ }^{24}$, l'analyste déduit leur emplacement dans le tronc et proposa une restitution de la grume (Fig. 7).

Les quatre planches étaient des paires de fausses-dosses, situées de part et d'autre de l'axe du tronc. La première et la troisième avaient du être sciées avant les deux autres, placées plus à l'intérieur de la grume. La restitution, i.e. l'image scientifique, permettait dans ces conditions de comprendre l'altération de l'image artistique. Les planches tirées des parties excentrées du tronc sont normalement plus sensibles aux variations thermohygrométriques que celles situées en profondeur. Or la troisième, où la conservatrice-restauratrice avait détecté des craquelures en saillie, était la plus excentrée des quatre. Le défaut de planéité du support et les altérations de la couche picturale pouvaient donc s'expliquer par la sensibilité particulière de cette planche, naturellement différente de celle de ses voisines.

\section{Raisonnement par cas}

17 Quelle nouvelle conclusion en tirer sur le rapport des images qui nous intéressent dans le champ de la conservation-restauration? Les experts les ont mobilisées dans le cadre d'un examen diagnostique pour constituer un cas singulier: celui du panneau de la Sainte-Anne, sans équivalent. Ce type d'inférence ne se réduit pas aux abductions et aux déductions qu'il enchaîne, mais n'est pas non plus un raisonnement par prototype, puisqu'il ne s'appuie pas sur des similitudes, mais sur des singularités. Il ne va pas de la règle au cas, comme la déduction, mais du fait au fait, sans inférer de règles, à la différence de l'induction. Ce n'est pas non plus un raisonnement «à partir » de cas, parce qu'il ne se réfère pas à d'autres, déjà traités et en établit plutôt un, unique en son genre. Tandis que l'abduction logique invoque une règle pour expliquer un fait, il conjecture une exception ${ }^{25}$. On peut appeler raisonnement "par» cas ce type d'inférence, distinct des cinq précédentes. Celui de la Sainte-Anne a pour particularité de mobiliser des images à la fois scientifiques et artistiques et de ne pas être fait par un seul individu, mais par un groupe d'experts dont les conjectures se renforcent pour définir un cas unique. Interprofessionnel et pluridisciplinaire, il partage certains traits caractéristiques avec le raisonnement clinique et la science forensique, qui appartiennent à la grande famille des raisonnements par cas. L'analogie entre la restauration et la médecine semble donc épistémologiquement fondée. Mais elle ne porte pas sur le but de ces disciplines, comme on pourrait le croire : il ne s'agit pas de dire que la première est aux œuvres ce que la seconde est aux hommes - i.e. un art de prolonger la vie ${ }^{26}$ - mais de montrer que leurs praticiens pensent de la même façon, c'est-à-dire par cas - par abduction, déduction ou prototype - pour faire un diagnostic à partir d'indices ou de symptômes. L'analogie porte sur les modes d'inférences et le raisonnement par cas ne sépare pas ici les professionnels qui interviennent dans ce champ ; il les réunit plutôt dans le paradigme indiciaire des médecins, des détectives et des historiens d'art, selon son inventeur Carlo Ginzburg ${ }^{27}$. 


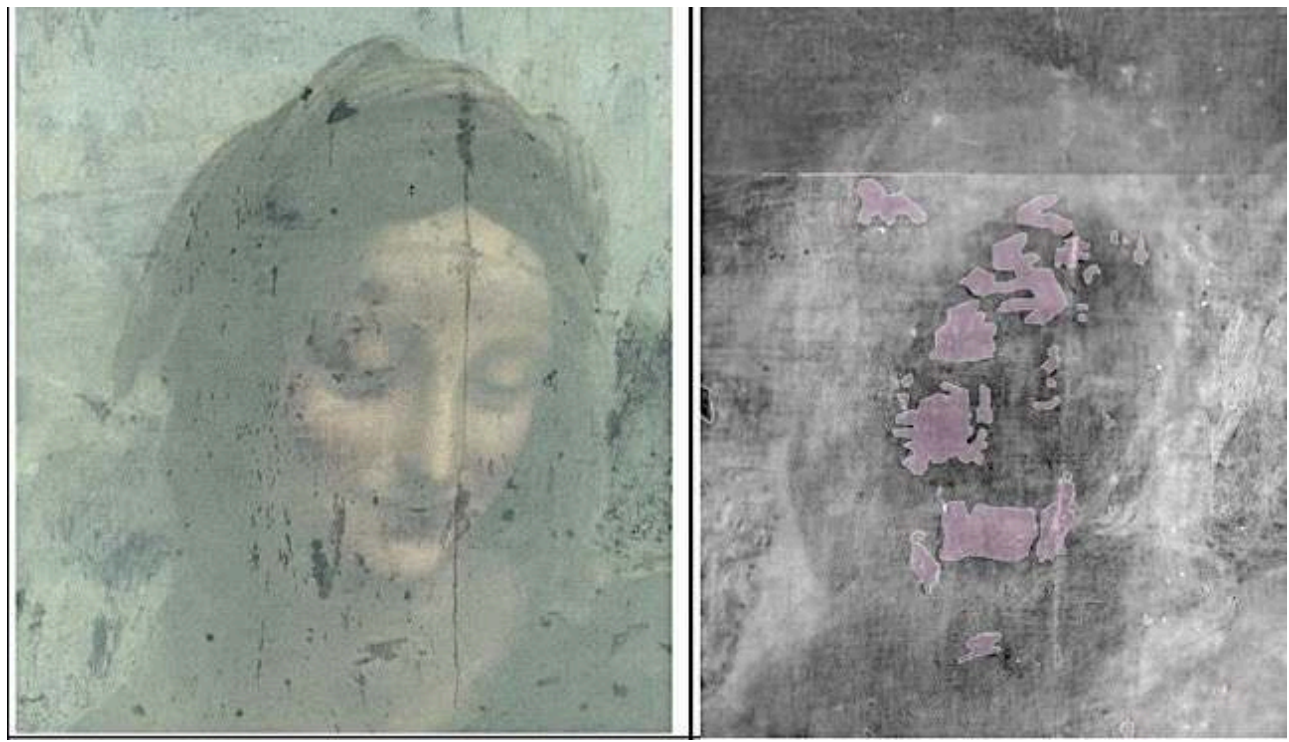

Visage de sainte Anne en ultraviolets et émissiographie (16279)

(c) $2 R M F$

Conçue comme une aide à la décision, l'imagerie scientifique servit dans le cas de la Sainte-Anne à confirmer les conjectures des experts ou à arbitrer leurs différends. La fluorescence d'ultraviolets mis en évidence les repeints entre les vernis et l'émissiographie montra les glacis et les lacunes de la couche picturale (Fig. 8).

La réflectographie infrarouge rendait visible le dessin sous-jacent, ainsi que les repentirs et les points du spolvero. Les rayons $\mathrm{X}$ traversaient la couche picturale jusqu'à la préparation et au support, dont ils découvraient la texture. Chaque longueur d'onde pénétrait plus profondément dans l'épaisseur de l'œuvre, où elle était absorbée, réfléchie ou diffractée selon le poids atomique des matériaux; la photographie objectivait leur présence, en captant leur image dans les franges éloignées du spectre électromagnétique. Ces clichés, qui rendaient pour ainsi dire "visible l'invisible", donnèrent lieu à des envolées lyriques et à des débordements métaphysiques auxquels le laboratoire de recherche dut en partie son succès médiatique ${ }^{28}$. Plus prosaïquement, ils permirent ici de dresser la carte des repeints et des lacunes de la couche picturale. L'analyse par spectrophotocolorimétrie, micro fluorescence X (MFX), fluorescence X (XRF) et diffraction X (XRD) permit de reconstituer la palette de Léonard et de cartographier les pigments. L'examen au microscope optique de quelques prélèvements de la couche picturale, analysés au microscope à balayage électronique (MEB-EDX), précisa sa stratigraphie. Tous ces résultats et ces images scientifiques devaient être interprétés pour produire des connaissances dans le champ de l'histoire de l'art et être utiles aux conservateurs chargés de superviser la restauration. Le problème posé par ce genre d'interprétation fut clairement formulé en 1929, lors de la création des premiers laboratoires dans les musées ${ }^{29}$. Il n'était pas sans rappeler celui du cercle herméneutique bien connu des philologues qui établissent et traduisent les textes anciens $s^{30}$. Sommairement : on ne pouvait interpréter correctement l'image scientifique qu'en ayant compris l'image artistique, mais on ne pouvait voir clairement une image artistique qu'en ayant bien interprété son image scientifique. La plupart des pays industrialisés firent de la conservation-restauration une discipline à part entière pour 
sauter dans ce cercle. Ils ouvrirent des écoles au carrefour de l'art et de la science, où les praticiens apprirent les langages qui leur permettent d'interpréter scientifiquement une image artistique ou d'utiliser l'imagerie scientifique dans une perspective patrimoniale ${ }^{31}$. Aucun professionnel ne pouvant prétendre maîtriser seul toutes ces connaissances, ces pays résolurent aussi de former des commissions interprofessionnelles pour suivre la restauration des chefs d'œuvres qu'ils tenaient pour un patrimoine commun de l'humanité32. Il en fut ainsi de la Sainte-Anne.

\section{Fantasme des spectres}

Figure 9

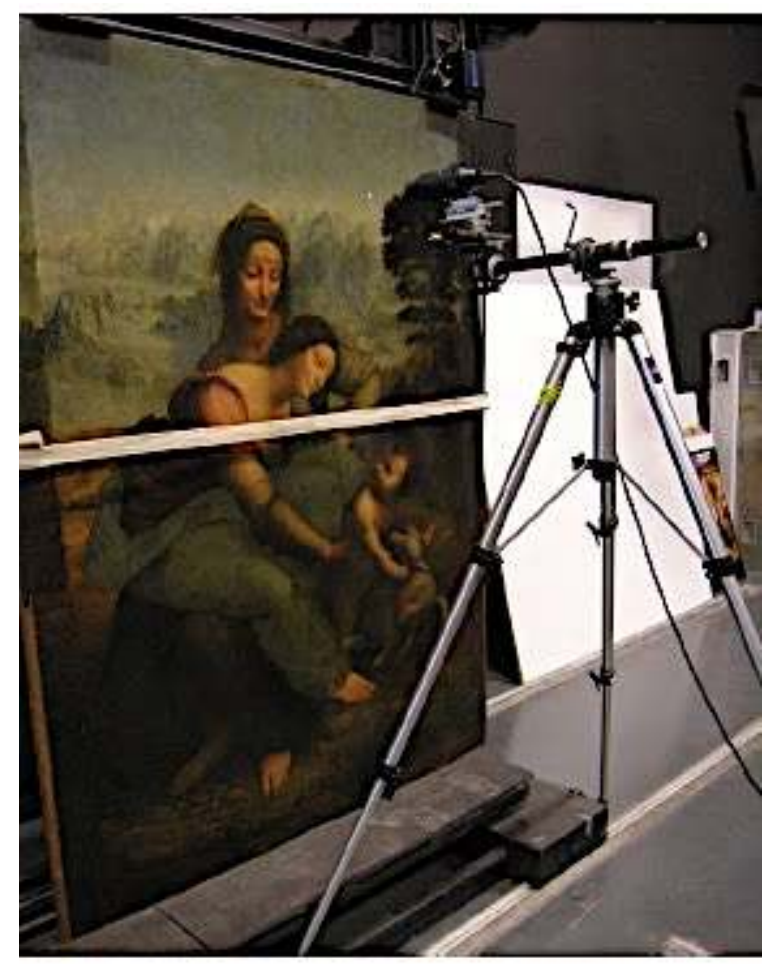

Spectrocolorimètre sans contact du C2RMF (16285)

(c) $2 \mathrm{RMF}$

Le laboratoire établit la carte des pigments par différentes techniques d'analyses chimiques: la micro-florescence X (MFX), la spectrophotocolorimétrie $\mathrm{L}^{*} \mathrm{a}^{*} \mathrm{~b}^{*}$, la fluorescence $X$ directe ou couplée à la diffraction X (XRD-XRF). Le principe consistait à bombarder de rayon $\mathrm{X}$ un point de l'œuvre et à analyser le rayonnement émis en retour. L'analyse du spectre d'émission permettait de déduire la composition des matériaux visés lorsque leur signature spectrale apparaissait, ou de la conjecturer dans un mélange quand leur courbe avoisinait celle caractérisant un élément connu. La spectrophotocolorimétrie $\mathrm{L}^{*} \mathrm{a}^{*} \mathrm{~b}^{*}$ utilise ce principe pour restituer les couleurs des matériaux dans le spectre du visible ${ }^{33} . L^{*}$ représente la clarté ; $a^{*}$ la proportion de rouge par rapport au vert ; $b^{*}$ celle du jaune par rapport au bleu. Ces mesures qui chiffrent la couleur dans l'espace chromatique (CIELAB) permirent aux experts du laboratoire, Clotide Boust, Jean-Jacques Ezrati et Myriam Eveno, de dresser en 2008 le constat d'état colorimétrique de la Sainte-Anne. Le spectrocolorimètre portable du C2RMF, hissé sur 
un trépied au niveau des points à mesurer, fonctionnait comme l'œil de Lyncée au sommet du mât de l'Argos, sondant la matière par son rayon visuel (Fig. 9).

Figure 10

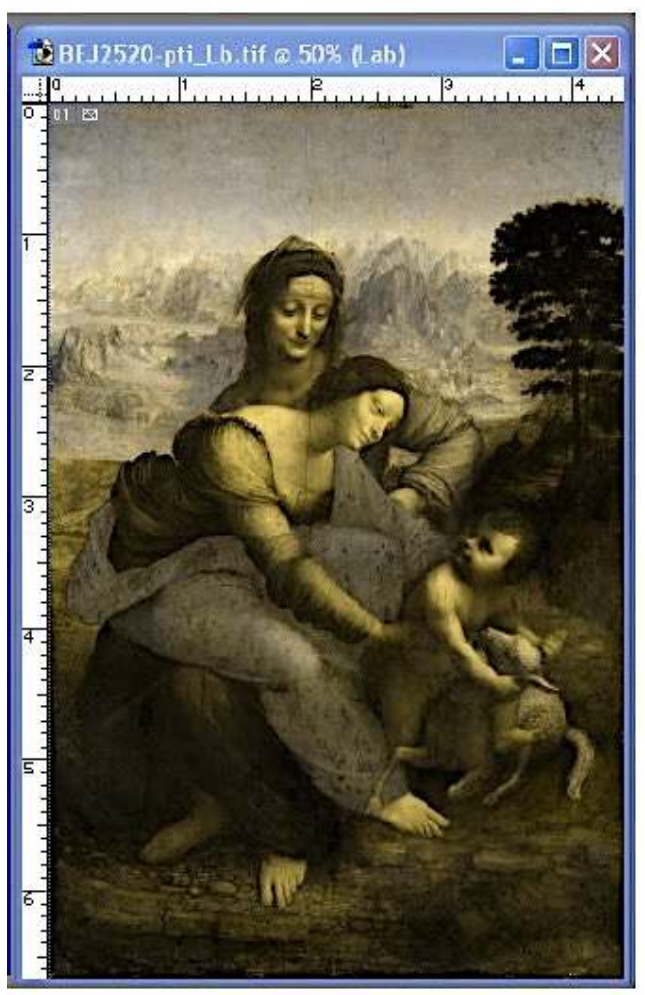

Images L*a et L*b de la Sainte-Anne (16285)

(C) $2 R M F$

Le croisement des résultats avec ceux obtenus par micro-fluorescence X (MFX) et examen au microscope électronique à balayage (MEB-EDX) permirent de cartographier les pigments et de reconstituer la palette de Léonard, puis de modéliser la superposition des couches picturales et d'en conjecturer l'exécution. Les experts proposèrent aussi d'utiliser les résultats de l'examen spectrophotocolorimétrique $\mathrm{L}^{*} \mathrm{a}^{*} \mathrm{~b}^{*}$ pour évaluer l'état chromatique de l'œuvre après restauration. L'intervention devait diminuer l'épaisseur du vernis jauni dont l'effet était immédiatement visible sur l'image L*b* du tableau avant l'opération (Fig. 10).

Le jaune couvrait toute la surface et écrasait le bleu du ciel, de l'eau et des montagnes au loin. L'image scientifique objectivait le filtre qui faussait la perception de l'image artistique et montrait ce que l'amincissement du vernis, c'est-à-dire la restauration, devait faire disparaître pour qu'apparaisse enfin ce qu'il cachait. 
Figure 11

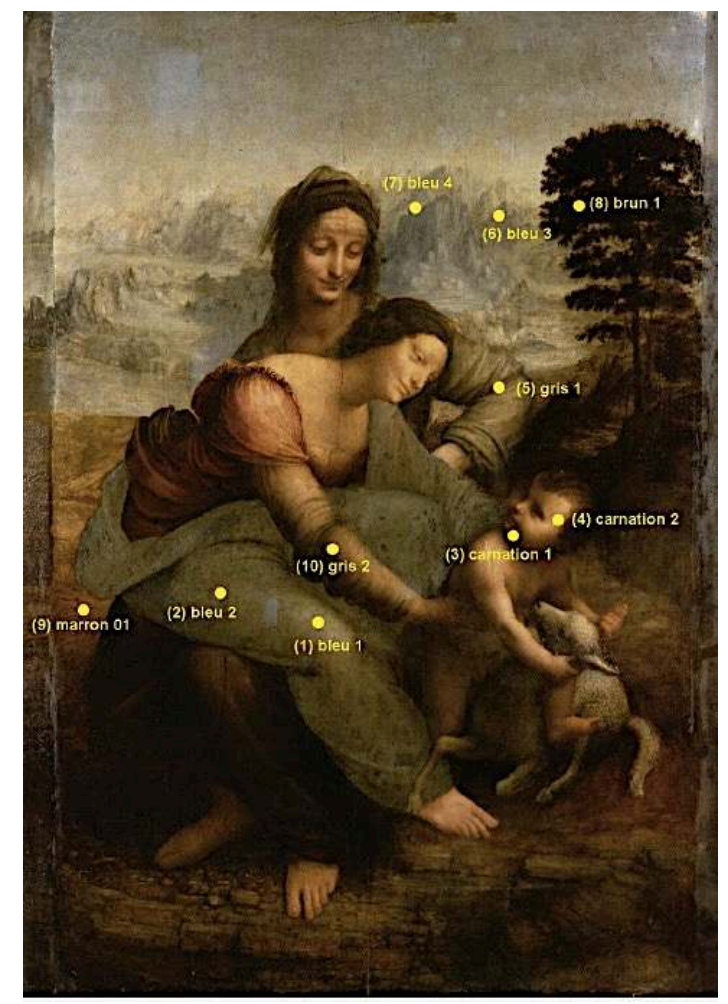

Points mesurés par diffraction X (16281)

(c) 2 RMF

Quelles données les experts échangèrent-ils pour opérer ce renversement qui révéla enfin l'œuvre? Les spectres de 122 points analysés sur l'ensemble de l'œuvre devaient la dévoiler. 35 furent mesurés par spectrophotocolorimétrie, regroupés par zones choisies suivant un dégradé de nuances ${ }^{34} ; 47$ par fluorescence $\mathrm{X}$, réalisés sur les carnations des trois personnages, le long de lignes ou isolement ${ }^{35} ; 28$ autres par microfluorescence X (MFX) pointés sur les couleurs de l'œuvre et répartis sur toute sa surface $^{36} ; 12$ enfin, par diffraction X (XRD), plus précisément ciblés sur les bleus, les bruns, les gris, les carnations et les marrons (Fig. 11) ${ }^{37}$. 
Figure 12

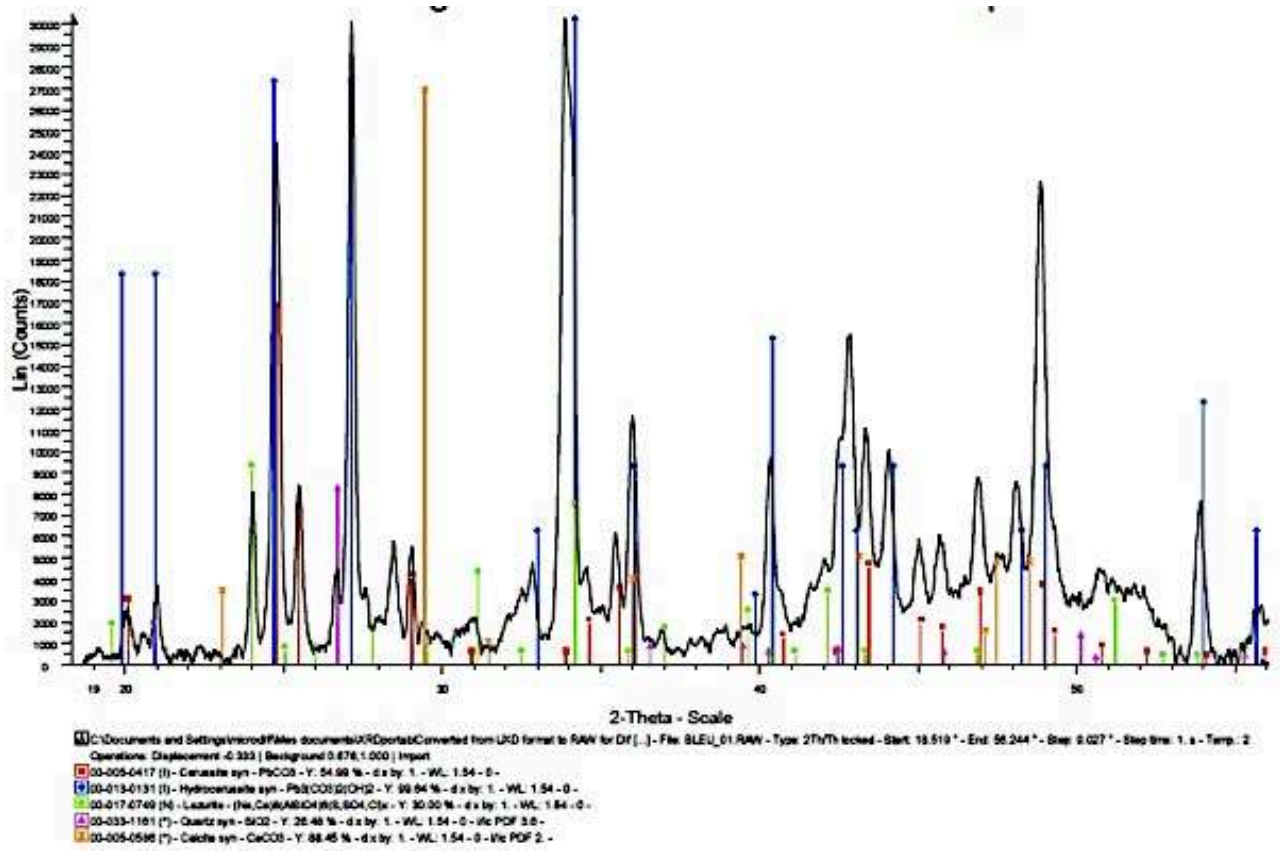

Mesure XFD du pointé 01, robe de la Vierge (16281)

(C) $2 R M F$

Les résultats des mesures se présentaient sous la forme de courbes spectrales, c'est-àdire de diagrammes de distribution d'énergie permettant d'identifier ou de conjecturer la présence d'éléments chimiques selon leurs signatures ou leurs traits caractéristiques (Fig. 12). 
Tableau (1/2) des analyses en MFX (tube en argent) Leonard de Vinci - La Vierge, l'Enfant Jésus et Saint Anne (F5950)

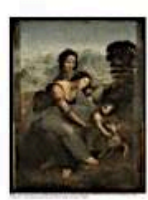

\begin{tabular}{|c|c|c|}
\hline $\begin{array}{c}\text { Numéro du pointé, couleur et } \\
\text { emplacement }\end{array}$ & Eléments détectés. ${ }^{1}$ & $\begin{array}{c}\text { Pigment principal, supposé, } \\
\text { à l'origine de la couleur. }{ }^{2}\end{array}$ \\
\hline 01. Brun de l'arbre à senestre. & $\begin{array}{l}\text { Plomb, cuivre, calcium, } \\
\text { fer, potassium, chlore, } \\
\text { nickel. }\end{array}$ & Vert au cuivre altéré. \\
\hline $\begin{array}{l}\text { 02. Bleu du ciel à la hauteur de la } \\
\text { cime de l'arbre à senestre. }\end{array}$ & $\begin{array}{l}\text { Plomb, calcium, } \\
\text { potassium, fer, silicitum. }\end{array}$ & Outremer: \\
\hline $\begin{array}{l}\text { 03. Bleu du ciel au-dessus du } \\
\text { pointé } 02 \text {. }\end{array}$ & $\begin{array}{l}\text { Plomb calcium, } \\
\text { potassium, fer, silicium, } \\
\text { altuminitum. }\end{array}$ & Outremer: \\
\hline $\begin{array}{l}\text { 04. Bleu des montagnes les plus } \\
\text { éloignées à droite de l'arbre à } \\
\text { senestre. }\end{array}$ & $\frac{\text { Plomb, calcium, }}{\text { potassium. fer, silicium. }}$ & Outremer. \\
\hline $\begin{array}{l}\text { 05. Bleu des montagnes les plus } \\
\text { claires à gauche de la tête de } \\
\text { Sainte Anne. }\end{array}$ & \begin{tabular}{|l}
$\begin{array}{l}\text { Plomb } \\
\text { potassium, fer, silicitum, } \\
\text { barvum. }\end{array}$ \\
\end{tabular} & Outremer: \\
\hline $\begin{array}{l}\text { 06. Carnation claire de la joue } \\
\text { droite de Sainte Anne. }\end{array}$ & $\begin{array}{l}\text { Plomb, fer, calcium, } \\
\text { potassium, mercure, } \\
\text { silicium. }\end{array}$ & $\begin{array}{l}\text { Mélange de blanc de plomb, } \\
\text { de vermillon et d'ocre rouge }\end{array}$ \\
\hline $\begin{array}{l}\text { 07. Carnation sombre du nez de } \\
\text { sainte Anne. }\end{array}$ & $\begin{array}{l}\text { Plomb, fer, calcium, } \\
\text { potassium, mercure, } \\
\text { manganese, silicium. }\end{array}$ & $\begin{array}{l}\text { Mélange de blane de plomb, } \\
\text { de vermillon et d'ocre rouge }\end{array}$ \\
\hline $\begin{array}{l}\text { 08. Bleu, repeint, à côté de l'épaule } \\
\text { droite de sainte Anne. }\end{array}$ & $\begin{array}{l}\text { Plomb, calcium, } \\
\text { potassium, fer, silicium, } \\
\text { barvum, manganèse, }\end{array}$ & $\begin{array}{l}\text { Outremer pour le bleu } \\
\text { original. }\end{array}$ \\
\hline
\end{tabular}

Tableau des résultats d'analyses en MFX (16284) (c) 2 RMF

L'équipe pluridisciplinaire chargée de cartographier les pigments et de reconstituer la palette du maître, transcrit ces données dans un tableau à trois colonnes: dans la première, les points mesurés étaient liés aux couleurs observées, aux éléments détectés dans le deuxième et aux pigments correspondants dans la troisième (Fig. 13).

Le tableau coordonnait ainsi les approches physicaliste et phénoméniste de l'œuvre, en même temps que les régimes iconographiques correspondants. Chacun renvoyait à des jeux de langages et à des pratiques très différentes. L'approche scientifique était "physicaliste", au sens où elle réduisait implicitement l'œuvre à ses composantes physico-chimique et l'appréhendait comme un objet matériel. L'approche artistique était en revanche " phénoméniste », parce qu'elle postulait que l'œuvre subsistait dans ce support et survenait dans l'esprit de ceux qui savaient l'y reconnaitre ${ }^{38}$. Objet physique pour les uns, intentionnel pour les autres, le « tableau » se dédoublait ainsi en entrant dans le champ de la conservation-restauration: à celui peint par l'artiste s'ajoutait celui des analystes qui répertoriaient les éléments détectés par microfluorescence ou diffraction $\mathrm{X}^{39}$; la " composition » était celle de la matière picturale dans l'un, mais celle des formes et des couleurs dans l'autre. Les "signatures» identifiaient des types physico-chimiques ou des individus réels et l'image obtenue était " artistique » ou " scientifique » selon la lumière, visible ou invisible, qui éclairait l'œuvre, le changement de régime iconographique s'expliquant physiquement par le déplacement de l'objet dans le spectre électromagnétique.

Dans le cas qui nous intéresse, ce dédoublement excluait cependant que l'imagerie scientifique puisse définir l'image artistique qui apparaîtrait finalement. Tous les pigments de la Sainte-Anne se décoloraient en effet avec le temps, chacun à son rythme 
en raison des échanges chimiques internes ou externes à l'œuvre ${ }^{40}$. Son état chromatique, cinq cent ans après sa création, n'était plus celui d'origine et l'on ne pouvait espérer retrouver ses couleurs en la restaurant, irrémédiablement passées avec le temps. Mais on ne pouvait pas non plus les refaire, en les déduisant de l'analyse des pigments, sous peine de fabriquer un faux historique. La règle d'or de la restauration interdisait de passer du "pigment à l'origine de la couleur», inscrit sur le tableau physicaliste de l'œuvre, à la " couleur du pigment à l'origine » connu des peintres et des conservateurs. Une telle restauration n'aurait produit qu'un fantasme scientifique au sens platonicien du terme : l'œuvre serait devenue la copie de l'image scientifique censée restituer son état d'origine. Parce que les choses que l'on produit d'après leur image sont des «fantasmes" - ou des fantômes (idole) dans la terminologie platonicienne ${ }^{41}$ - on pourrait appeler «fantasme des spectres » celui qui consiste à imaginer que la restitution spectrométrique d'une œuvre puisse servir de modèle ou guider sa restauration. Le dévernissage virtuel de la Mona-Lisa donne une idée assez aboutie de ce type de fantasme, qui fait de l'image artistique un sous-produit de l'image scientifique $^{42}$.

Comment restaurer la Sainte-Anne dans ces conditions ? Quelle image en donner ${ }^{43}$, si les couleurs cachées sous le vernis jauni n'étaient plus celles voulues par Léonard et s'il était exclu d'en restituer l'éclat d'origine? Le principe de l'altération des pigments avec le temps étant admis, la solution du Musée du Louvre consistait traditionnellement à donner au vernis protecteur le rôle de filtre chromatique ${ }^{44}$. Héritier des travaux de Diderot sur la balance de la composition, René Huyghe, qui dirigea le Département des peintures durant une quinzaine d'années, avait défendu avec le directeur italien de l'Institut central de restauration, Cesare Brandi, le principe de l'allègement "nuancé » des vernis lors de la querelle sur le nettoyage des peintures, qui opposa les Latins aux Anglo-Saxons partisans d'un dévernissage plus poussé ${ }^{45}$. La patine des vernis pouvait, selon Huyghe et Brandi, atténuer la disharmonie chromatique due au passage du temps et rétablir le délicat équilibre des anciennes compositions, à jamais perdu. La restauration du dernier chef-d'œuvre de Léonard allait-elle confirmer la règle? Comment dévernir la Sainte-Anne: fallait-il ôter uniformément sa couche protectrice, apocryphe et jaunie? Ou l'amincir à certains endroits, pour ôter les repeints et maintenir l'équilibre du tout? Quelle main pouvait s'y risquer? On dit que l'histoire se répète deux fois. De fait, la querelle des vernis connut un nouvel épisode en France, soixante ans après son déclenchement en 1947 à Londres.

\section{Bascule de point}


Figure 14

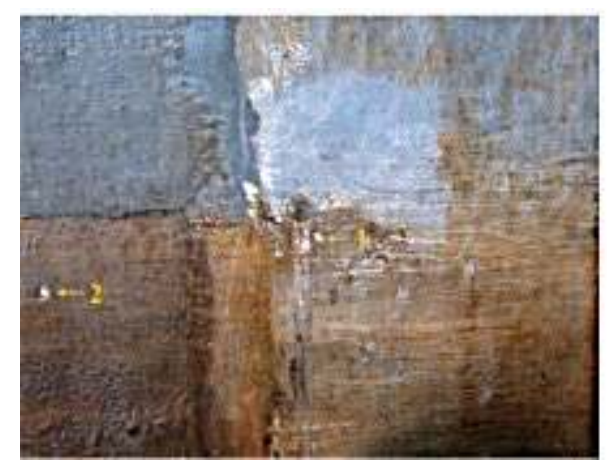

Test d'amincissement (25151)

(c) 2 RMF

La conservatrice-restauratrice chargée de l'opération, Cinzia Pasquali, fit d'abord quelques tests de dévernissages sur des zones marginales ou problématiques du tableau. On appelle ces tests des "fenêtres ", parce qu'elles en ont la forme et sont plus transparentes que le vernis ôté (Fig. 14).

Ces ouvertures correspondent à un degré d'amincissement et dévoilent en partie l'image artistique. Elles montrent ce que serait le tableau si l'on généralisait l'opération et fonctionnent comme des «avant/après » en miniature. Au premier plan, en surface, on peut voir l'œuvre avant l'opération et par la fenêtre, en perspective ou arrière-plan, ses couleurs après restauration. Le vernis jauni qui encadre la partie amincie présente l'échantillon comme un petit tableau dans un grand. Son sujet, son studium, n'est cependant plus celui de l'œuvre dans son ensemble : ce n'est pas l'histoire de la SainteAnne et de l'Enfant Jésus, ni même un détail de ce récit. C'est celle de la fabrication de l'image passée et présente avec, en perspective, sa transmission à venir : l'historia sur laquelle s'ouvre la fenêtre de dévernissage est son histoire matérielle, inscrite dans l'épaisseur de la couche picturale ${ }^{46}$. La dynamique des plans n'a pas échappé au réalisateur du documentaire sur la restauration de «l'ultime chef d'œuvre» de Léonard. Comment a-t-il filmé le changement de régime iconographique qui nous intéresse ? Comment basculer continuellement d'un point de vue à l'autre, de l'art à la science et du phénoménisme au physicalisme? L'œil des experts fonctionnerait-il comme une caméra dont le point focal se déplace durant l'action? Victor Stoichita, dont les travaux sur la fenêtre en ont inspiré d'autres sur l'imagerie scientifique ${ }^{47}$, a déjà exploré la piste que l'on se propose de suivre ${ }^{48}$.

\section{Tests de d'amincissement}




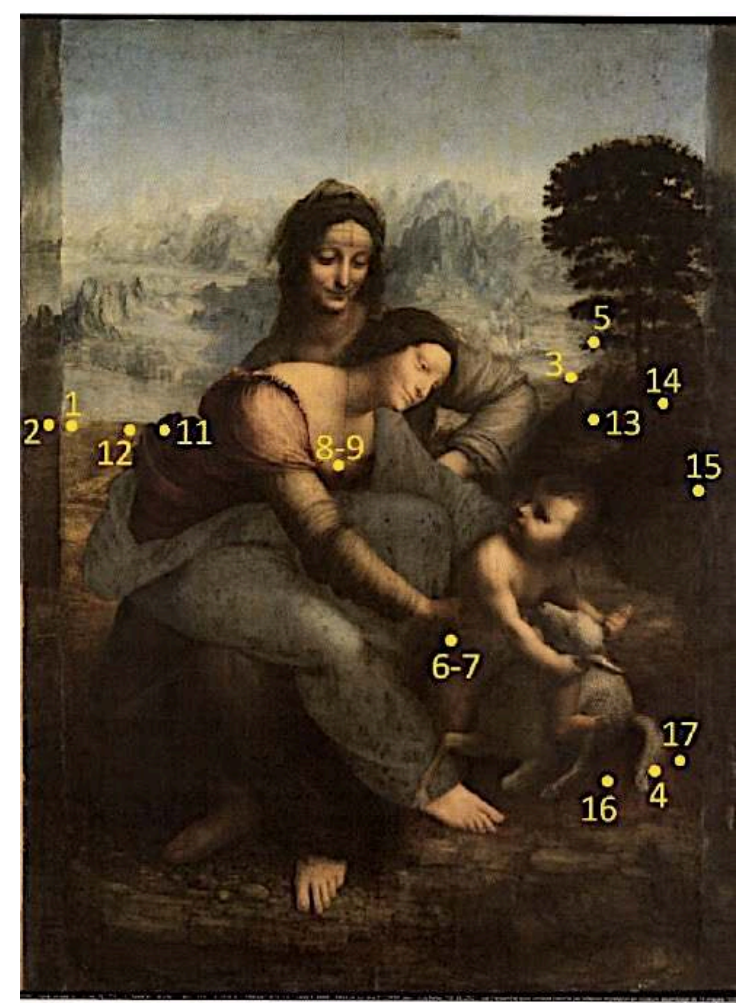

Localisation des prélèvements de 2012 (25151)

cC2RMF

31 Dans le cas présent, la validation des tests de dévernissage suivit un protocole strict. Leurs résultats furent d'abord soumis aux membres de la commission internationale formée pour l'occasion, qui réunissait des conservateurs, des restaurateurs et des historiens d'art ayant pour rôle de conseiller le maître d'œuvre de l'opération. Il appartenait à ce dernier, après délibération, soit de demander des examens complémentaires pour répondre aux questions que posait le diagnostic de la commission, soit de valider le niveau d'amincissement testé sur une zone donnée et de l'étendre aux voisines, sinon à l'ensemble de l'œuvre. Le laboratoire intervenait, dans le premier cas, pour effectuer les analyses demandées ou pour mesurer, dans le second, l'épaisseur du vernis aminci. Le premier type d'intervention donna lieu en $2008^{49}$ et en $2012^{50}$ à une vingtaine de prélèvements effectués directement sur la couche picturale, examinés au microscope optique puis électronique à balayage (MBE). La cartographie de cette nouvelle série de punctum localise les points d'interrogation de la Commission sur l'état de l'œuvre (Fig. 15). 
Figure 16

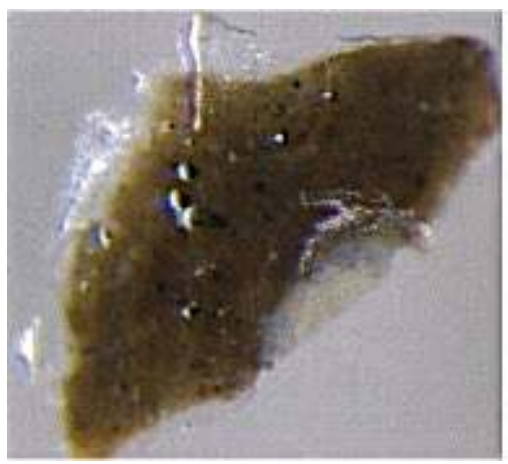

Échantillon 1 (25151)

(c) $2 R M F$

Le laboratoire fut parfois autorisé à répondre à ses questions par une analyse chromatographique de quelques échantillons ${ }^{51}$. Dans les autres cas, les prélèvements furent d'abord enrobés d'une résine de maintien, puis taillés perpendiculairement et polis pour éliminer les rayures (Fig. 16).

Figure 17

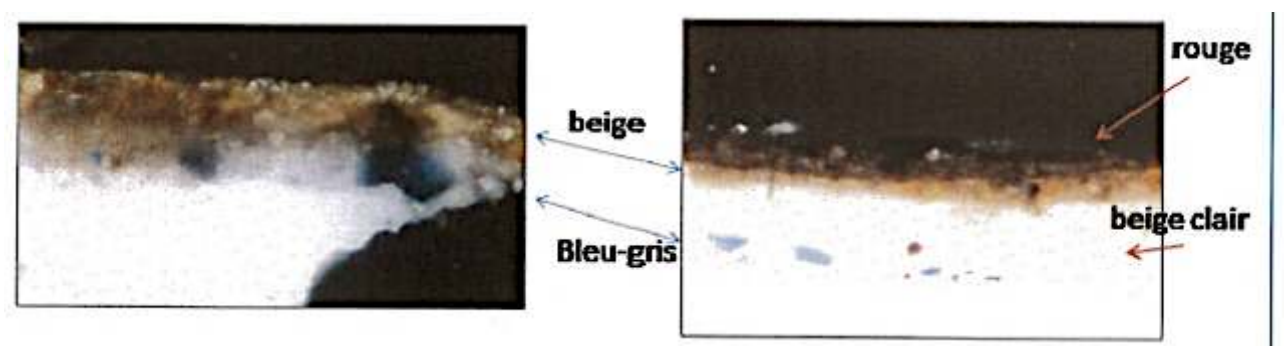

Échantillon 12 et 11 (25151)

(c) $2 R M F$

33 Les analystes pouvaient ainsi observer au microscope les sections obtenues et analyser leurs composantes, pour reconstituer l'histoire matérielle de l'œuvre en se basant sur le principe stratigraphique, selon lequel les couches les plus profondes sont plus anciennes que les suivantes. Découvrant le temps dans l'espace, ils purent ainsi répondre aux questions de la Commission, en expliquant par exemple que la couche beige du troisième plan du tableau, après les bleus du ciel et des montagnes, était authentique parce qu'on la retrouvait aussi sous les rouges de la robe de la Vierge, ellemême originale (Fig. 17).

L'histoire que ces microprélèvements permettaient de reconstruire n'était pas celle de la Sainte-Anne, mais de la fabrication de l'œuvre. Les coupes stratigraphiques étaient les indices matériels qui permirent aux analystes de remonter de la matière à l'image artistique par inférences successives. Invisibles à l'œil nu, ces points de Lyncée fabriqués par la science n'étaient observables qu'au microscope. À la différence des soulèvements de la couche picturale, détectés par la conservatrice-restauratrice lors du constat d'état, ils ne conduisaient pas le regard de la surface à la profondeur, mais lui frayaient un chemin inverse, depuis les strates sous-jacentes jusqu'aux modelés apparents. Opposés par leur orientation, ces points de Lyncée articulaient comme les précédents les deux régimes iconographiques qui nous intéressent, en opérant un va et 
vient entre l'art et la science. Mais comment cette bascule et ces croisements se firentils réellement : comment les experts parvinrent-ils à articuler leurs points de vue et à raisonner ensemble? Le documentaire sur la restauration de la Sainte-Anne en donne une idée, forcément plus artistique que scientifique ou logique.

\section{Fantasme des studies}

Figure 18

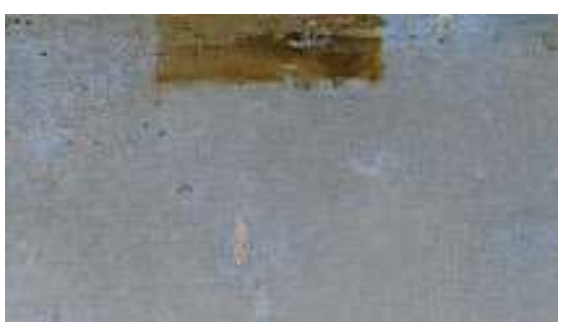

Témoin de dévernissage (27380)

(c) $2 \mathrm{RMF}$

Avant amincissement complet, le documentaire montre au premier plan le vernis jauni qui encadre la fenêtre, alors que l'image artistique paraît en arrière-plan. Mais la perception de leur position relative s'inverse à l'issue de l'opération. L'image artistique apparaît au premier plan, tandis que le témoin jauni conservé en marge du tableau passe à l'arrière-plan. Or cette technique d'inversion a un nom au cinéma : c'est la «bascule de point » (rack focus). La caméra passe en continu de l'avant à l'arrière-plan en se focalisant sur une zone lointaine pour suivre une action. Ce qui paraissait flou et sans relief devient net par déplacement du point focal tandis que la scène située au premier plan bascule dans l'indistinction. De même, l'amincissement du vernis met au point l'image artistique, au sens où elle permet à l'œil de se focaliser sur son modelé plutôt que sur les irrégularités du vernis. L'opération, extrêmement lente et séquencée, n'est évidemment pas la même qu'au cinéma, mais fait aussi apparaître, par déplacement du point focal, les reliefs et la distinction des plans que floutaient l'épaisseur de la couche protectrice. Elle rend aussi à l'image la profondeur de champ que le vernis écrasait. Ce qui ne pourrait être qu'une analogie fut une réalité pour la conservatrice-restauratrice intervenant sur la couche picturale et pour les membres de la commission qui suivirent ses travaux. Amincir la couche jaunie et faire réapparaître l'image imposait à la praticienne une bascule continue du point focal de son regard d'un sujet à l'autre: de l'image patinée située à l'avant-plan, à l'image nettoyée émergeant à l'arrière-plan, ou de l'histoire matérielle de l'œuvre à celle que représentait la scène, d'un studium à l'autre. De même, les membres de la commission, qui assistaient à l'intervention tandis que les deux états coexistaient encore, devaient redoubler d'attention pour juger du résultat en effectuant à leur tour cette même opération, qui faisait du studium lui-même, c'est-à-dire de l'histoire, le punctum visé. En plaçant le modelé de l'image artistique au premier plan et en réduisant le vernis jauni à un témoin d'arrière-plan, la restauration acheva la bascule et stabilisa l'image. Ces deux histoires de l'œuvre - l'une matérielle, l'autre représentée - sont aujourd'hui visibles sur deux plans différents : la composition de Léonard sur le premier et le témoin de l'intervention en marge du tableau (Fig. 18). 

agglutinés dans les vernis du manteau de la Vierge pour les broderies dont ils venaient d'apprendre l'existence en cours; ni comme Freud, Jung ou Pfister qui virent dans ce manteau dont Léonard n'avait pas achevé le modelé, une image cryptée justifiant leur diagnostic $^{52}$. Ces interprétations, également erronées ${ }^{53}$, relevait d'un même biais de confirmation consistant à projeter dans le tableau son sujet d'étude à partir d'une reconnaissance vague, d'une rémanence ou d'une paréidolie. On pourrait appeler « phantasme des studies » cette tendance à réinterpréter des objets, sinon le monde, en fonction de son studium pour renouveler une approche, avec plus ou moins de succès.

Que conclure de ce nouvel épisode sur la relation des images artistiques et scientifique dans le champ de la conservation-restauration? La bascule de point opère ici entre l'histoire matérielle de l'œuvre et la scène représentée, c'est-à-dire entre deux studii. L'un focalise le regard sur le vernis à amincir, d'un point de vue scientifique et physicaliste, tandis que l'autre le porte sur les modelés à faire réapparaître, c'est-à-dire sur l'image artistique. Le déplacement du point focal du regard qui suit l'action articule dynamiquement les deux régimes iconographiques que les points de Lyncée reliaient successivement. Ces derniers sont les "points de bascule " de la «bascule de point » dynamique où l'œil des experts oscille d'un sujet à l'autre, de la surface à la profondeur et de l'image artistique à son corrélat scientifique. Comment les experts ont-ils opéré cette mise au point et se sont-ils finalement entendus?

\section{Points de contrôle}

Figure 19

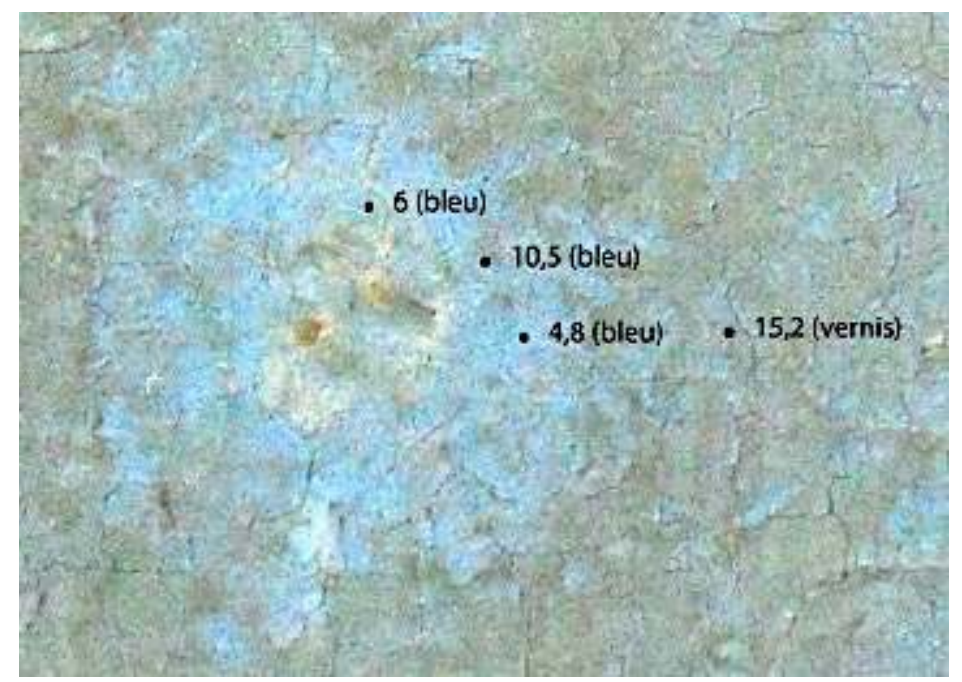

Différences d'aspect entre les parties allégées et très allégées du ciel (27396) (c) $2 R M F$

L'épaisseur du vernis avait été mesurée en 7 points du tableau, dès son arrivée au laboratoire, grâce à une station de micromesure optique ${ }^{54}$. En 2008, cinq cent ans après sa création et une dizaine de restaurations, elle était en moyenne de 25 microns. Son amincissement faisait courir un risque à l'œuvre, dont la couche picturale pouvait être touchée et dégradée ${ }^{55}$. Les compétences de la conservatrice-restauratrice minimisaient ce risque et les conservateurs demandèrent au laboratoire de remesurer régulièrement 
l'épaisseur du vernis pour s'assurer de l'innocuité du traitement. Ce contrôle devait aussi servir à arbitrer objectivement les discussions que l'opération ne manquerait pas de susciter au sein de la Commission, car le poids de la querelle des vernis, qui cliva les Latins et les Anglo-Saxons après-guerre, pesait encore sur la restauration. Dès la première réunion, le conservateur Pierre Curie, alors responsable de la filière peinture au Centre de restauration, pointa la contradiction qui allait raviver l'ancienne querelle. On ne pouvait en effet demander à la conservatrice-restauratrice de dégager les repeints en régularisant seulement le vernis, puisqu'il fallait amincir ce dernier pour atteindre les premiers, ce qui créerait des irrégularités ${ }^{56}$. Moins elle allégerait cette couche, plus elle devrait retoucher le tableau pour masquer les repeints dont les couleurs avaient virées, tandis que plus elle l'amincirait, moins elle aurait à le retoucher. Lors de sa deuxième réunion, la commission opta pour un allègement très modéré du vernis, conforme aux règles de prudence et de progressivité qu'elle s'était donnée ${ }^{57}$. Mais elle constata à sa troisième que certains tests, poussés plus avant par la conservatrice-restauratrice, rendaient mieux le modelé de l'image artistique que les

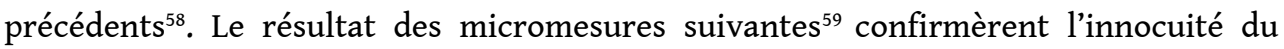
procédé et son rendu esthétique plaida en faveur de la proposition de la praticienne. La commission choisit dans ces conditions de réviser sa position, suivant le principe de progressivité qui la guidait. Elle autorisa, lors de sa quatrième réunion, la conservatrice-restauratrice à descendre au-dessous du seuil des 18 microns sur les zones qu'elle avait jusqu'alors sanctuarisées, quitte à rompre avec la doctrine héritée de René Huyghe ${ }^{60}$. À la demande des maîtres d'œuvre de l'opération, Jean-Jacques Ezrati effectua en septembre 2011 une quarantaine de nouvelles mesures sur des points situés dans sept zones sensibles du tableau ${ }^{61}$. Pour chacune, l'analyste dressa le profil de la couche de vernis et définit la clarté de l'image en chaque point selon le niveau d'amincissement. Dans le cas des bleus - dont la distinction des plans dépendait en grande partie - les résultats montraient qu'ils paraissaient verdâtres à $13 \mu \mathrm{m}$, mais retrouvaient leurs couleurs à $8 \mu \mathrm{m}$ et semblaient éclatants à $5 \mu \mathrm{m}$ (Fig. 19). 


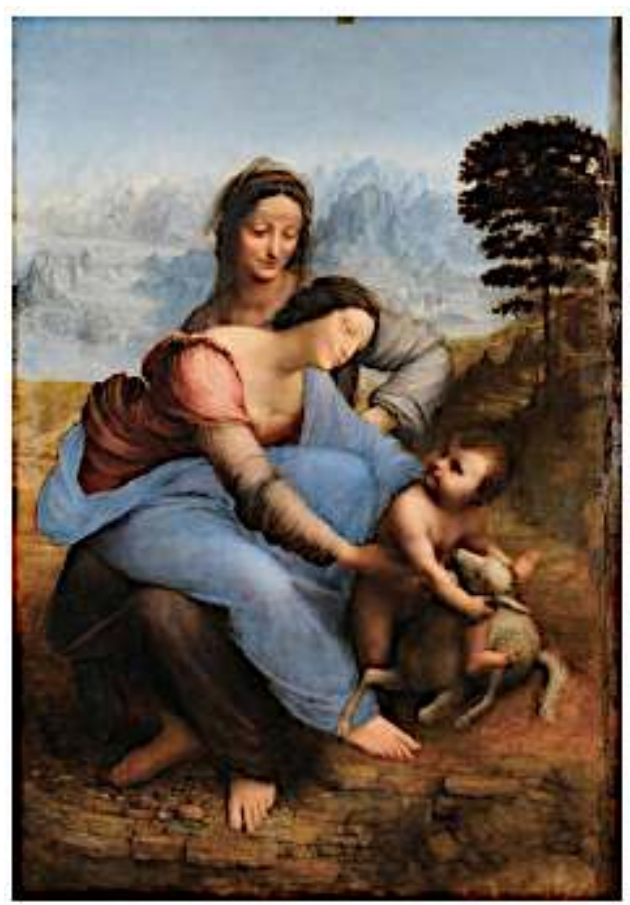

Tableau après restauration (27380)

(c) $2 R M F$

L'imagerie scientifique confirma ainsi la proposition de traitement que la conservatrice-restauratrice avait faite avant l'opération ${ }^{62}$. Les clichés montraient objectivement à tous les membres de la Commission ce qu'elle découvrait en restaurant l'œuvre. En s'appuyant sur ces analyses et sur un jugement esthétique, couplée à une règle de restauration selon laquelle on ne retouche pas une retouche, la commission trancha enfin lors de sa cinquième réunion le dilemme formulé par Pierre Curie à la première ${ }^{63}$. On ne pouvait dégager les repeints, tout en régularisant le vernis, qu'en effectuant un allègement poussé et non modéré. Rien ne l'interdisait, puisque la couche amincie ne contenait aucun glacis ${ }^{64}$. La conservation de l'œuvre l'imposait même : ne pas descendre à ce niveau d'amincissement aurait obligé la restauratrice à masquer les anciens repeints par de nouvelles retouches, c'est-à-dire à réitérer le processus auquel il fallait mettre fin pour retrouver l'image. La commission se rendit à cet argument lors de sa dernière réunion et conclut l'affaire en rappelant qu'elle n'avait pas dérogé aux principes de progressivité et de sélectivité de l'amincissement que ses membres avaient unanimement adoptés ${ }^{65}$. L'exposition de «L'ultime chef-d'œuvre » de Léonard en mars 2012 montre le résultat de ce travail [Fig. 20].

\section{Conclusion}

Qu'en conclure finalement sur le rapport des images artistiques et scientifiques dans le champ qui nous intéresse? L'état d'une œuvre peut justifier le recours à l'imagerie scientifique, dans le cadre d'un examen diagnostique qui justifie à son tour une proposition de traitement, puis une intervention. Si cette dernière modifie l'image du bien, comme c'est généralement le cas lors d'une restauration, l'opération peut se réitérer tant que ses maîtres d'œuvre en ont les moyens, car cette modification est une 
altération, au bon ou au mauvais sens du terme, qui justifie comme précédemment le recours à l'imagerie scientifique. Entre le raisonnement expérimental et le syllogisme pratique, ce mode de penser non monotone est si sensible au contexte qu'il semble difficilement modélisable. Dans le cas de la restauration de la Sainte-Anne, on a vu qu'il articule deux régimes iconographiques et mobilise plusieurs modes d'inférences, dont l'abduction, la déduction, le raisonnement à partir de cas et le raisonnement par prototype. Le raisonnement expérimental à finalité pratique qui les réunit est collectif et non individuel, interprofessionnel autant qu'interdisciplinaire. Il pose la question de la coordination des points de vue et de l'enchaînement des inférences des experts impliqués dans le processus. On a analysé ce raisonnement par cas en montrant que la comparaison du chirurgien au restaurateur est épistémologiquement fondée, puis en élaborant le concept de "point de Lyncée » pour expliquer comment on bascule d'un régime iconographique à l'autre. Entre le fantasme des spectres et celui des études, chaque expert travaille dans son domaine, artistique ou scientifique, sur le sujet (studium) de l'autre et peut basculer dans son régime iconographique en focalisant son attention sur un point de l'image (punctum) qui renforce, modifie ou élimine les hypothèses du raisonnement expérimental à finalité pratique qu'ils font ensemble. Le modèle dynamique obtenu sur la base d'un seul cas n'a qu'une valeur heuristique. Il montre en l'occurrence que l'imagerie scientifique ne définit ni ne guide la restauration, mais la contrôle a posteriori et que l'amincissement du vernis met au point l'image artistique.

\section{NOTES}

1. ECCO, Compétences requises pour l'accès à la profession de conservateur-restaurateur, Ecco, 2013 [ Consulté le 1/1/2020: http://www.ecco-eu.org/fileadmin/assets/documents/publications/ ECCO_Competences_FR.pdf]

2. Vincent DELIEUvin (dir.), La Sainte Anne, l'ultime chef-d'œuvre de Léonard de Vinci, Musée du Louvre, Paris, 2012.

3. Stan neumann, Léonard de Vinci. La restauration du siècle, Arte / Musée du Louvre, 2012.

4. Stéphane LoIRE, «Le service d'Étude et de Documentation du département des Peintures du musée du Louvre », Documenter les collections des musées, Paris, La documentation Française, 2014, p. 75-92.

5. Christian LAHANIER, Geneviève AïTKEN, Marie-Thérèse BERGER, Catherine CHEVILlOT, Christophe PINCEMAILLE, «L'enjeu de la documentation au C2RMF », Techné n¹3, 2001 p. 27-34.

6. Clémence RAYNAUD, «Les archives de la restauration au Centre de recherche des musées de France ", Techné n'27, 2008, p. 42-45.

7. C2RMF, F 5950/41399, «Rapport d'intervention sur le support», Daniel Jaunard, Patrick Mandron, janvier 2012.

8. C2RMF, F5950/16279, «Compte-rendu d'étude C2RMF n4/13. Rapport provisoire », Élisabeth Ravaud, Paris, 20 octobre 2008. 
9. Élisabeth MARTin, Cécile scAillierez, Patrick LE ChANT, Jean-Paul Rioux, Nathalie volle, «La Vierge, L'Enfant Jésus et sainte Anne de Léonard de Vinci, Création et transmission d'un chef d'œuvre ", Techné n²1, 2005, p. 26-35.

10. C2RMF, F 5950/16288, «Cadre général de l'étude », Compte-rendu d'étude $n^{\circ} 1 / 13$, Bruno Mottin, 31 octobre 2008.

11. Vincent DELIEUVIN (dir.), La Sainte Anne, op. cit., p.215.

12. Ibid., p. 46 , note 4 .

13. Léonard DE VINCI : Étude de tête de cheval, RL 12327 ; Étude de crâne, RL 19058, Windsor Castle.

14. Stéphane LOIRE, « Le service d'Étude... », op. cit.., p. 81-83.

15. C2RMF F 5950/16278, «Identification de la technique graphique des dessins du revers », Alain Duval, 1 octobre 2008.

16. Catherine ALLAMEL-RAFFIN, «Un exemple d'étude comparée des procédures interprétatives à l'œuvre dans les sciences de la nature et dans l'analyse des œuvres d'art ", Arts et sciences. Approches sémiotiques et philosophiques des images, Presses Universitaires de Liège 2014, p. 107-122, notes 5 et 11.

17. Vincent DELIEUVIN (dir.), La Sainte Anne, op. cit. p. 46-200.

18. C2RMF, F 5950/16289, «Rapport d'étude. Examen direct du tableau et imagerie photographique (lumières directes et rasantes, réflectographies infrarouge, photographies de fluorescence sous ultraviolets) », Bruno Mottin, Jean-Louis Bellec, Elsa Lambert, 31 octobre 2008.

19. ICOM-CC, Terminologie de la conservation-restauration du patrimoine culturel matériel, New-Delhi, XVe Conférence Triennale, 22-26 Septembre 2008 (https://journals.openedition.org/ceroart/ 2795? file=1).

20. C2RMF, F 5950/16287, « Étude dendrochronologique des deux traverses au dos en sapin (Abies alba) du panneau peint », Catherine Lavier, Paris, 31 octobre 2008.

21. C2RMF, F 5950/8780, « Constat d'état », Nathalie Le Dantec, Paris, 28 mars 2006.

22. Daniel ARASSE, Le détail. Pour une histoire rapprochée de la peinture, Paris, Flammarion, 1992.

23. Lilly KAHIL, «Lynkeus et Idas », Lexicon iconographicum mythologiae classica, Zürich, Artemis \& Verlag, 1992, VI, 1, 319-322; 2, p. 165-166.

24. Odile CORTET, "Quelques aspect de conservation et de restauration au musée Magnin», Conservation et restauration, Dossier du département des peintures n²7, Paris, RMN, 1983, p.6-24.

25. Pierre LIVET, "Les diverses formes du raisonnement par cas", Penser par cas, Paris, EHESS, 2005, p. 229-253.

26. Camillo вогто, Conserver ou restaurer, Paris, L'imprimeur, 2000.

27. Carlo GINZBURG, "Traces. Racines d'un paradigme indiciaire», Mythes, emblèmes, traces. Morphologie et histoire, Lagrasse, Verdier, 1989, p. 218-294.

28. Madeleine HOURS, Les secrets des chefs-d'œuvre, Paris, Robert Laffont, 1964.

29. André BLUM, "Quelques méthodes d'examen scientifiques des tableaux et objets d'art", Mouseion, $\mathrm{n}^{\circ} 7,1929$, p.14-26.

30. Paul PHILIPPOT, « Réflexions sur le Problème de la Formation des Restaurateurs de Peintures et de Sculptures ", Studies in Conservation, Vol. 5, N², Mai 1960, p. 61-70.

31. Pierre LEVEAU, L'institution de la conservation du patrimoine dans l'Entre-Deux-Guerres, Dijon, OCIM/Universitaires de Bourgogne, 2017.

32. Paul COREMANS, L'Agneau mystique au Laboratoire. Examen et traitement, Anvers, De Sikkel, 1953, p. 7-10.

33. C2RMF, F 5950/16285, «Compte-rendu d'étude C2RMF n¹1/13, Analyses spectrophotocolorimétriques ", Clotilde Boust, Jean-Jacques Ezrati, Myriam Eveno, Paris, 30 octobre 2008. 
34. C2RMF, F 5950/16285, «Compte-rendu d'étude C2RMF n¹1/13, Analyses spectrophotocolorimétriques ", Clotilde Boust, Jean-Jacques Ezrati, Myriam Eveno, Paris, 30 octobre 2008.

35. C2RMF, F 5950/16283, "Compte-rendu d'étude C2RMF n¹0/13, Étude des carnations par fluorescence X », Philippe Walter, Éric Laval, Laurence de Viguerie, Paris, 29 octobre 2008.

36. C2RMF, F 5950/16284, "Compte-rendu d'étude C2RMF $n^{\circ} 8 / 13$, Micro fluorescence $X$ portable », Éric Laval et Jean-Louis Bellec, Paris, 30 octobre 2008.

37. C2RMF, F 5950/16281, "Compte-rendu d'étude C2RMF $n^{\circ} 9 / 13$, Diffraction des rayons X, Fluorescence X, XRD-XRF », M. Eveno, J. Castaing et A. Duran, Paris, 27 octobre 2008.

38. Pierre LEVEAU, «La querelle des vernis et le différent des sciences et des lettres ", Synergies Royaume-Uni et Irlande, $\mathrm{n}^{\circ}$, 2014, p. 57-69 (https://gerflint.fr/Base/RU-Irlande7/leveau.pdf).

39. C2RMF, F5950/16280 : "Compte-rendu d'étude C2RMF, Étude de la palette », Myriam Eveno, Paris, 27 octobre 2008.

40. Ségolène BERGEON LANGLE, «L'Éloge du doute en Restauration des œuvres d'art », Mélanges en l'honneur de Roger Marijnissen, CeRoart, HS 2018 (https://journals.openedition.org/ceroart/4627).

41. PLATON, République, $\mathrm{X}, 596 \mathrm{~d}-598 \mathrm{~d}$.

42. Pascal cotTE, Mona Lisa dévoilée. Les vrais visages de la Joconde, SW Télémaque, 2017.

43. C2RMF, F 5950/-, « Numérisation multispectrale ", Compte-rendu d'étude n $13 / 13$, Geneviève Aiken, Philippe Colantoni, Ruven Pillay, 2 novembre 2008.

44. Grazia NICOSIA, «Le vernis des apparences. Incidences visuelles et cognitives du nettoyage des tableaux ", CeRoart n5, 2010 (https://journals.openedition.org/ceroart/1483).

45. UNESCO : Le traitement des peintures, Paris, Museum, Vol.III, $\mathrm{n}^{\circ} 2-3,1950$.

46. Philippe WALTER, Sur la palette de l'artiste. La physico-chimie dans la création artistique, Collège de France-Fayard, 2014.

47. Maria Giulia DONDERO, «Voir en art, voir en science », Nouvelle revue d'esthétique, $2016 \mathrm{n}^{\circ}$ 17, PUF, p. 139-159.

48. Victor stoichita, L'effet Sherlock Holmes, Paris, Hazan, 2015.

49. C2RMF, F5950/16282, "Compte-rendu d'étude C2RMF $n^{\circ} 7 / 13$, Microscopie électronique à balayage - Étude de pigments », Laurence de Viguerie et Myriam Eveno, Paris, 27 octobre 2008.

50. C2RMF, F 5950/25151, «Compte-rendu d'étude n²5151, Étude en cours de restauration Chromatographie en phase liquide », Myriam Eveno, Witold Nowik et Jean-Louis Bellec, Paris, 31 août 2012.

51. C2RMF, F 5950/24462, «Rapport d'étude n²4462, Étude des vernis », Élisabeth Ravaud, Myriam Eveno et Sigrid Mirabaud, Paris, 11 avril 2012.

52. Sigmund FREUD, Un souvenir d'enfance de Léonard de Vinci, Paris, Gallimard, 1977.

53. Vincent DELIEUVIN (dir.), La Sainte Anne, op. cit., p.342-343 et 357.

54. C2RMF, F 5950/16286, "Rapport de mesures d'épaisseurs effectuées sur Saint Anne de Léonardo de Vinci », Jean-Jacques Ezrati, Christel Doublet, Clotilde Boust, 29 septembre 2008.

55. Ségolène BERGEON, Restauration des peintures, Dossier du département des peintures $n^{\circ} 21$, Paris, RMN, 1980, p. 9-25.

56. C2RMF, F 5950/26588, "Commission internationale de restauration de la Sainte-Anne de Léonard de Vinci », première réunion, 18 juin 2010.

57. C2RMF, F 5950/26589, "Commission internationale de restauration de la Sainte-Anne de Léonard de Vinci », deuxième réunion, 25 janvier 2011.

58. C2RMF, F 5950/26590, "Commission internationale de restauration de la Sainte-Anne de Léonard de Vinci », troisième réunion, 19 avril 2011.

59. C2RMF, F 5950/27395, « Mesure de l'épaisseur du vernis », Jean-Jacques Ezrati, Clotilde Boust, 2 mars $2011 ; 22688$ : «Mesure de l'épaisseur du vernis (suivi) demandée en février 2011 par le comité », Jean-Jacques Ezrati, Vilma Fraize, Estelle Itié, 14 avril 2011; 22688: "Mesure de 
l'épaisseur du vernis demandée en mai 2011 par la réunion du comité du 27 juin 2011 », JeanJacques Ezrati, Estelle Itié, 8 juin 2011.

60. C2RMF, F 5950/26592, "Commission internationale de restauration de la Saint Anne de Léonard de Vinci », quatrième réunion, 27 juin 2011.

61. C2RMF, F 5950/27396, "Étude demandée en juillet 2011 par le département des peintures du musée du Louvre pour la réunion du comité de septembre 2011 », Jean-Jacques Ezrati, 2 Septembre 2011.

62. C2RMF, F 5950/41389, "Mémoire méthodologique. Restauration du tableau La Vierge, l'Enfant Jésus et Saint Anne », Cinzia Pasquali, 2011.

63. C2RMF, F 5950/26594, "Restauration de la Sainte-Anne de Léonard de Vinci », Commission scientifique du 3 janvier 2012.

64. C2RMF, F 5950/21861, « Analyse PIXE », Laurent Pichon, Myriam Eveno, 7 février 2011.

65. C2RMF, F 5950/26594, "Restauration de la Sainte-Anne de Léonard de Vinci », Commission scientifique du 16 février 2012.

\section{RÉSUMÉS}

Il existe au moins un domaine où les images scientifiques et artistiques sont similaires. C'est celui de la conservation-restauration des biens culturels. À partir d'une étude de cas, on montre que les analogies faites dans ce domaine par les historiens d'art ne sont pas les conclusions, mais des prémisses : ce sont les indices des raisonnements abductifs qu'ils soumettent aux scientifiques du patrimoine dans le cadre des raisonnements expérimentaux à finalité pratique qu'ils font ensemble. Les conservateurs-restaurateurs qui interviennent sur l'œuvre sont en position d'articuler ces deux perspectives, entre l'art et la science. On identifie dans cet article les points de bascule d'un régime iconographique à l'autre et l'on modélise cette bascule de point, en empruntant au cinéma quelques concepts. Les résultats de cette étude de cas devront être confrontés à d'autres, pour livrer aux experts de la conservation-restauration une sémiotique qui leur soit utile.

There is at least one field where scientific and artistic images are similar. It is that of the conservation-restoration of cultural property. From a case study, it is shown that the analogies made in this field by art historians are not conclusions, but premises: they are the indicators of the abductive reasoning which they submit to heritage scientists in the framework of experimental for practical purposes that they make together. The conservators involved on the work are in position to articulate these two perspectives, between art and science. We identify in this article the tipping points from one iconographic regime to another and we model this rack focus, borrowing a few concepts from cinema. The results of this case study will have to be compared with others, in order to deliver semiotics to the conservation experts.

\section{INDEX}

Mots-clés : patrimoine, restauration, abduction, indice, Léonard de Vinci

Keywords : heritage, restoration, abduction, symptom, Leonardo da Vinci 


\section{AUTEUR}

\section{PIERRE LEVEAU}

Pierre Leveau est docteur en philosophie et membre associé aux recherches du Centre Gilles Gaston Granger (UMR 7304). Il enseigne la philosophie dans le secondaire et a soutenu une thèse de doctorat à Aix-Marseille Université sur l'épistémologie de la conservation et de la restauration du patrimoine culturel matériel (ED 356). Qualifié aux sections 17 et 72 du CNU en 2013, il a publié en 2017 la partie historique de son travail aux Presses universitaires de Dijon et poursuit actuellement ses recherches dans une série d'articles sur la métaphysique et la sémiotique de la conservation-restauration $(\mathrm{CRBC})$. Il s'intéresse pour cela plus particulièrement à l'héritage sémiotique de C. Pierce, au paradigme indiciaire de C. Ginzburg et à la forensique. L'article sur la Saint-Anne de L. de Vinci est le premier d'un triptyque, dont les deux autres porteront sur la Joconde et Saint Jean-Baptiste. 